\title{
Genetic analysis of praziquantel response in schistosome parasites implicates a transient receptor potential channel
}

\author{
Le Clec'h Winka ${ }^{1,{ }^{*}}$, Chevalier Frederic D. ${ }^{1}$, Mattos Ana Carolina A. ${ }^{2}$, Strickland Amanda ${ }^{1}$, \\ Diaz Robbie ${ }^{1}$, McDew-White Marina ${ }^{1}$, Rohr Claudia M. ${ }^{3}$, Kinung'Hi Safari ${ }^{4}$, Allan Fiona ${ }^{5,6}$, \\ Webster Bonnie L. 5, 6, Webster Joanne P. 5, 7, Emery Aidan M. 5, 6, Rollinson David 5, \\ Djirmay Amadou Garba 8, 9, Al Mashikhi Khalid M. ${ }^{10}$, Al Yafae Salem ${ }^{10}$, Idris Mohamed A. ${ }^{11}$, \\ Mone Helene ${ }^{12}$, Mouahid Gabriel ${ }^{12}$, Loverde Philip ${ }^{2}$, Marchant Jonathan S. ${ }^{3}$, \\ Anderson Timothy J. C. ${ }^{1, *}$
}

\footnotetext{
1 Texas Biomedical Research Institute; San Antonio, TX USA

2 University of Texas Health Science Center at San Antonio; San Antonio, TX 78229, USA

3 3Department of Cell Biology, Neurobiology and Anatomy, Medical College of Wisconsin; Milwaukee WI 53226, USA

${ }^{4}$ National Institute for Medical Research, Mwanza, United Republic of Tanzania, Tanzania.

${ }^{5}$ London Centre for Neglected Tropical Disease Research (LCNDTR), Imperial College, London, United Kingdom, England.

${ }^{6}$ Wolfson Wellcome Biomedical Laboratories, Natural History Museum, London, United Kingdomn, England.

${ }^{7}$ Centre for Emerging, Endemic and Exotic Diseases (CEEED), Royal Veterinary College, University of London, United Kingdom, England.

${ }^{8}$ Réseau International Schistosomiases Environnemental Aménagement et Lutte (RISEAL), Niamey, Niger

9 World Health Organization, Geneva, Switzerland

$1^{0}$ Directorate General of Health Services, Dhofar Governorate, Salalah, Sultanate of Oman

$1{ }^{1}$ Sultan Qaboos University, Muscat, Sultanate of Oman

12 Host-Pathogen-Environment Interactions laboratory, University of Perpignan, Perpignan, France
}

* Corresponding authors : Winka Le Clec'h, email address : winkal@txbiomed.org ; Timothy J. C. Anderson, email address : tanderso@txbiomed.org

\begin{abstract}
:
Mass drug administration with praziquantel (PZQ) monotherapy is considered the mainstay for control and elimination of the parasites causing schistosomiasis in humans. This drug shows imperfect cure rates in the field, and parasites showing reduced PZQ response can be selected in the laboratory, but the extent of resistance in Schistosoma mansoni populations is unknown. We examined the genetic basis of the variation in response in a PZQ-selected S. mansoni population (SmLE-PZQ-R) in which 35\% of the parasitic worms survive high-dose PZQ (73 micrograms per milliliter) treatment. We used genome-wide association to map loci underlying $\mathrm{PZQ}$ response and identified a transient receptor potential (Sm.TRPMPZQ) channel (Smp_246790) within the major chromosome 3 peak that is activated by nanomolar concentrations of PZQ. The PZQ response showed recessive inheritance and marker-assisted
\end{abstract}


selection of parasites at a single Sm.TRPMPZQ SNP that produced populations of PZQ-enriched resistant (PZQ-ER) and $P Z Q$-enriched sensitive (PZQ-ES) parasites, exhibiting $>377$-fold difference in $P Z Q$ response. The $P Z Q-E R$ parasites survived treatment in rodents at higher frequencies compared with PZQ-ES, and resistant parasites exhibited 2.25-fold lower expression of Sm.TRPMPZQ relative to sensitive parasites. Specific chemical blockers of Sm.TRPMPZQ enhanced PZQ resistance, whereas Sm.TRPMPZQ activators increased sensitivity. We surveyed Sm.TRPMPZQ sequence variations in 259 parasites from different global sites and identified one nonsense mutation that resulted in a truncated protein with no PZQ binding site. Our results demonstrate that Sm.TRPMPZQ underlies variation in PZQ responses in S. mansoni and provides an approach for monitoring emerging PZQ-resistant alleles in schistosome elimination programs. 


\section{INTRODUCTION}

Praziquantel (PZQ) is the drug of choice for treating schistosomiasis, a snail vectored parasitic disease, caused by flatworms in the genus Schistosoma. Schistosomiasis is widespread: three main parasite species infect over 140 million people in Africa, the Middle-East, South America and Asia (1, 2), resulting in widespread morbidity - a global burden of 1.9 million disability adjusted life years (3) - and mortality estimates ranging from 20 to 280 thousand annually $(4,5)$. Pathology results from eggs that lodge in the liver and intestine (S. mansoni and S. japonicum) or in the urogenital system (S. haematobium) stimulating granuloma formation. This results in a spectrum of pathology including portal hypertension, hepatosplenic disease, bladder cancer, genital schistosomiasis and infertility. S. mansoni infection alone results in a conservative estimate of 8.5 million cases of hepatosplenomegaly in sub-Saharan Africa (6). Mass drug administration programs currently distribute an estimated 250 million doses of PZQ per year aimed in the short term at reducing schistosome associated morbidity and mortality, and in the longer term at eliminating schistosomiasis transmission $(7,8)$. PZQ is also widely used for treatment of other flatworm parasites of both humans and livestock including tapeworms.

PZQ treatment of adult worms results in rapid $\mathrm{Ca}^{2+}$ influx into cells, muscle contraction and tegument damage (9-12). Both the mechanism of action and the mechanism of resistance to PZQ have been the focus for much speculation and research $(13,14)$. Several proteins like calcium gated channels $(15-17)$ or ABC transporters $(18,19)$ have been suspected to play a role in PZQ resistance. However, this topic has been stimulated by the recent finding that a transient receptor channel $\left(S m . T R P M_{P Z Q}\right)$ is activated by nanomolar quantities of PZQ $(20,21)$.

Mass drug treatment with PZQ has enormous health benefits and has been extremely effective in reducing parasite burdens and transmission (8), but imposes strong selection for resistance on treated schistosome populations. Emergence of PZQ resistance is a major concern, because it could derail current progress towards WHO goal of eliminating schistosomiasis as a public health problem by 2025 (8). Several lines of 
evidence from both the field and the laboratory suggest that PZQ response varies in schistosome populations (22-27). PZQ resistance is readily selected in the laboratory through treatment of infected rodents or infected intermediate snail hosts (28). This typically results in a modest change (3-5 fold) in PZQ response in parasite populations $(28,29)$, although the PZQ resistance status of individual worms comprising these populations is unknown. PZQ treatment typically results in $\sim 30 \%$ of patients who remain egg positive following PZQ treatment (30). PZQ kills adult worms, but not immature parasites (31, 32), so both newly emerging adult parasites and drug resistance may contribute to treatment failure. There have been several reports of patients who remained egg positive across multiple PZQ treatment cycles (33, 34): schistosome infections established in mice from infective larvae from these patients showed elevated resistance to PZQ $(24,35)$. In Kenya and Uganda, infected communities where prevalence and disease burden are not reduced by repeated treatment have been identified. The causes of these "hotspots" $(36,37)$ is currently unknown, but PZQ-resistant schistosomes are one explanation. In a large longitudinal study of individual school age children, egg reduction ratios (ERR) were high in naïve populations treated with PZQ, but showed a significant decline after multiple rounds of treatment (38), consistent with selection of tolerance or resistance to PZQ. Identification of molecular markers for direct screening of levels of PZQ resistance alleles would be extremely valuable for parasite control programs, because changes in schistosome ERR have both genetic and non-genetic explanations and are laborious to measure.

The availability of good genome sequence and near complete genome assembly (39) for S. mansoni make unbiased genome wide approaches feasible for schistosome research (40). Our central goal is to determine the genetic basis determinants of variation in PZQ response, using genome wide association approaches. We exploit the PZQ-resistant parasites generated by laboratory selection (26) to determine the genetic basis of PZQ, identifying a transient receptor channel as the cause of variation in PZQ response. The Transient Receptor Potential Melastatin (TRPM) ion channel identified is activated by PZQ $(20,21)$ and has been designated Sm.TRPM $P Z Q$. Together, our genetic analysis and the independent pharmacological analysis by 

evolution in schistosome control programs.

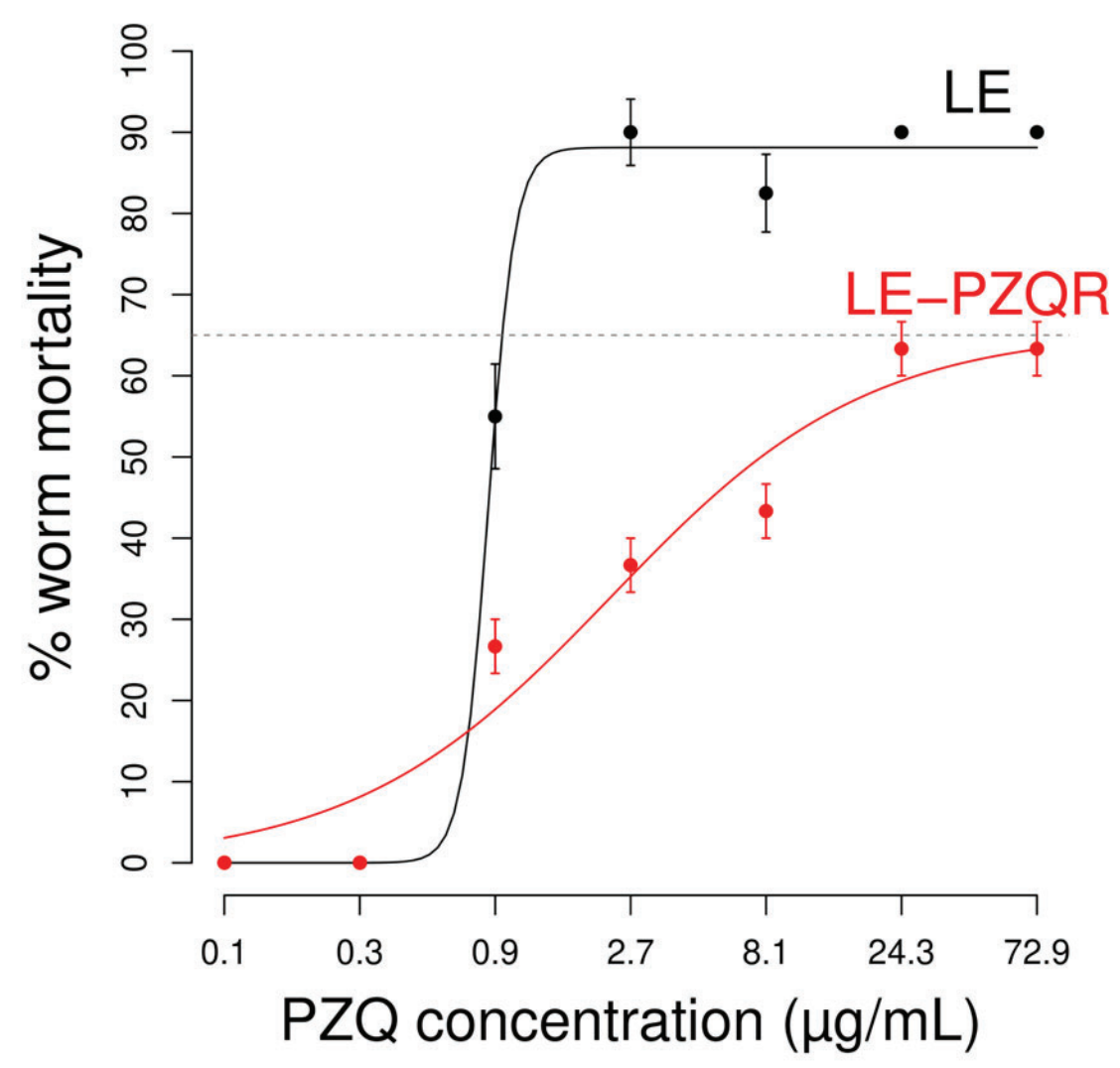

Fig. 1. Dose response curves for SmLE (PZQ-S) and the derived SmLE-PZQ-R (PZQ-R) populations. PZQ dose response curves show a $\sim 14$-fold difference in response between SmLE (ancestral population) and SmLE-PZQ (PZQ selected population) $\left(\chi^{2}\right.$ test $\left.=10.387, \mathrm{p}=0.001\right)$. The PZQ-selected laboratory schistosome population (LE-PZQ-R) is polymorphic for drug response. 35\% of SmLE-PZQ-R are not killed by treatment with high dose of PZQ, suggesting that this population is polymorphic ( $N=240 \mathrm{worms} /$ parasite populations). 
Male and female schistosome parasites pair in the blood vessels and reproduction is obligately sexual, so schistosomes are maintained in the laboratory as outbred populations. Hence, individual parasites within laboratory populations may vary in PZQ response. We measured PZQ response in the LE-PZQ-R population, which was previously generated by PZQ treatment of infected snails (26). This revealed a 14fold difference in $\mathrm{IC}_{50}$ between the LE progenitor parasite population $\left(\mathrm{IC}_{50}=0.86 \pm 0.14 \mu \mathrm{g} / \mathrm{mL}\right)$ and LEPZQ-R ( $\left(\mathrm{IC}_{50}=12.75 \pm 4.49 \mu \mathrm{g} / \mathrm{mL}, \chi^{2}\right.$ test, $\left.\mathrm{p}=0.001\right)$ derived by PZQ selection (Fig. 1). This is higher than the 3-5 fold differences observed between PZQ-selected and unselected parasite populations in previous studies (28). Interestingly, the dose response curve for the LE-PZQ-R population plateaus at $65 \%$ mortality: the remaining $35 \%$ of parasites recovered even at high dose of PZQ $(72.9 \mu \mathrm{g} / \mathrm{mL})$. These results suggest that the LE-PZQ-R parasite population is a mixed population that contains both PZQ-sensitive and PZQresistant individual worms (Fig. 1).

\section{Association mapping of PZQ resistant genes identifies a TRPM channel}

We conducted a genome wide association study (GWAS) to determine the genetic basis of PZQ resistance (PZQ-R). GWAS has been widely used for mapping drug resistance in parasitic protozoa (41) and the model nematode Caenorhabditis elegans (42), but has not previously been applied to parasitic helminths, because of the difficulty of accurately measuring drug response in individual parasites. When worms are treated with PZQ, there is a massive influx of $\mathrm{Ca}^{2+}$ into cells and parasites contract $(17,43)$, but some worms recover and resume respiration and movement $24-48 \mathrm{~h}$ after drug removal. We assayed parasite recovery following high dose PZQ treatment $(24 \mu \mathrm{g} / \mathrm{mL})$ of individual male worms maintained in 96 -well plates by measuring L-lactate production (44), a surrogate measure of respiration, 48h after PZQ treatment removal (Fig. S1). These assays allow efficient measurement of recovery in individual PZQ-treated worms.

We conducted replicate experiments $(A: n=590 ; B: n=691)$ to measure PZQ response in individual parasites maintained in 96-well plates. The distributions of L-lactate production in the two experiments 

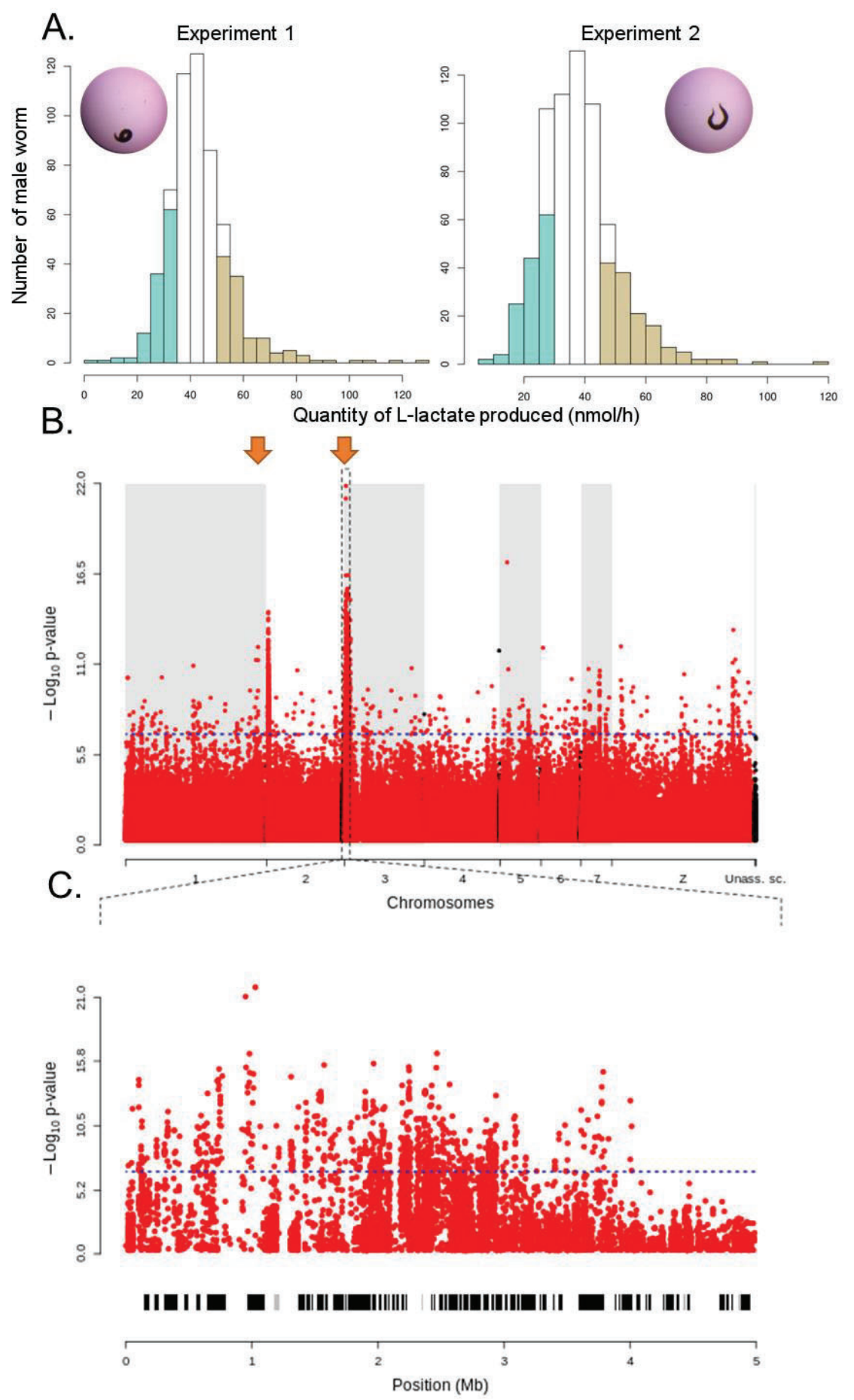

Fig. 2. Genome-wide association mapping of PZQ response. (A). We measured recovery of individual adult male worms following expose to $24 \mu \mathrm{g} / \mathrm{mL}$ PZQ by measuring lactate production. The distribution from both experimental replicates is shown (A: $N=590$; B: $N=691$ ). Worms in the bottom and top quintile were each pooled, and genome sequenced to high read depth. (B). The Manhattan plot identifies genome regions that differ in allele frequency between high and low lactate worm pools. (C). The chr. 3 QTL identified spans $4 \mathrm{Mb}$ and 91 genes, including several promising candidates. 
were broad (A: 0-126.56 nmol/h, mean $=42.95 \mathrm{nmol} / \mathrm{h}$; B: 0-118.61 nmol $/ \mathrm{h}$, mean $=37.79 \mathrm{nmol} / \mathrm{h})$ : we identified worms from the top and bottom quintile for lactate production (Fig. 2A) which were then bulk sequenced to high read depth (average read depth - A: 39.97; B: 36.83). Two genome regions (chr. 2 and chr. 3) showed strong differentiation in allele frequencies between parasite populations showing high and low lactate production phenotypes (Fig. 2B). The highest peak $\left(\mathrm{p}=1.41 \times 10^{-22}\right)$ on chr. 3 spanned $4 \mathrm{Mb}$ $(22,805-4,014,031 \mathrm{bp})$ and contained 91 genes, of which 85 are expressed in adult worms (Fig. 2C). This genome region contains several potential candidate loci including three partial $\mathrm{ABC}$ transporters (Table S1). One gene close to the highest association peak is of particular interest: Smp_246790 is a transient receptor potential channel in the M family (TRPM). This same channel was recently shown to be activated by PZQ following exposure to $\mathrm{nM}$ quantities of drug resulting in massive $\mathrm{Ca}^{2+}$ influx into HEK293 cells transiently expressing this protein $(20,21)$. This gene, designated $S m \cdot T R P M_{P Z Q}$, is therefore a strong candidate to explain variation in PZQ response within parasite populations. Two other features of the data are of interest. First, the SNP (position $1029621 \mathrm{~T}>\mathrm{C}$ ) marking the highest association peak (at 1,030 Mb) is found in a transcription factor (Smp_345310, SOX13 homology) from a family known to regulate splicing variants (45). Second, there is a $\sim 100 \mathrm{~kb}$ deletion (1220683- $1220861 \mathrm{bp)} 6.5 \mathrm{~kb}$ from Sm.TRPMPZQ and another $150 \mathrm{~kb}$ deletion $(1,200,000-1,350,000 \mathrm{bp}) 170 \mathrm{~kb}$ from the transcription factor. This was enriched in high lactate groups in both replicates and is in linkage disequilibrium with the enriched SNP in Sm.TRPMPZQ.

The chr. 2 peak $\left(\mathrm{p}<1.0 \times 10^{-15}\right)$ spans $1.166 \mathrm{Mb}(291,191-1,457,462 \mathrm{bp})$ and contains 24 genes $(21$ expressed in adult worms). This genome region contains no genes that could be a candidate to explain variation in PZQ response. 
To confirm these associations and determine whether the loci underlying PZQ response are inherited in a

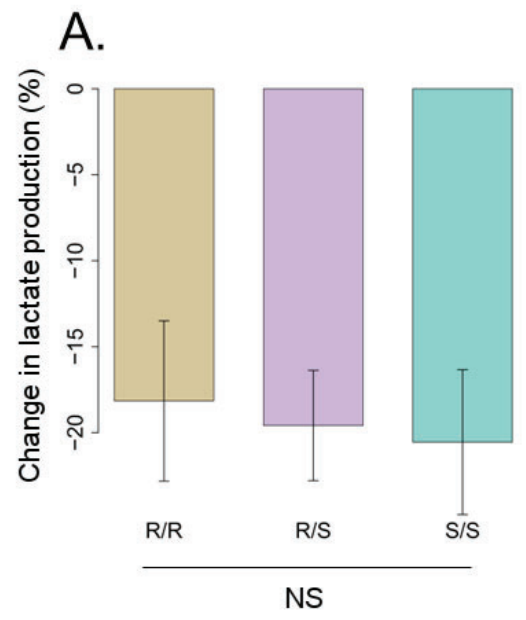

B.

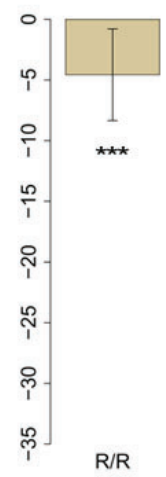

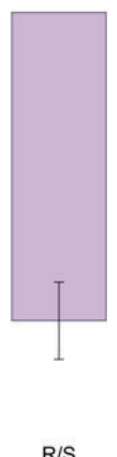

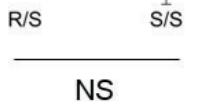

C.

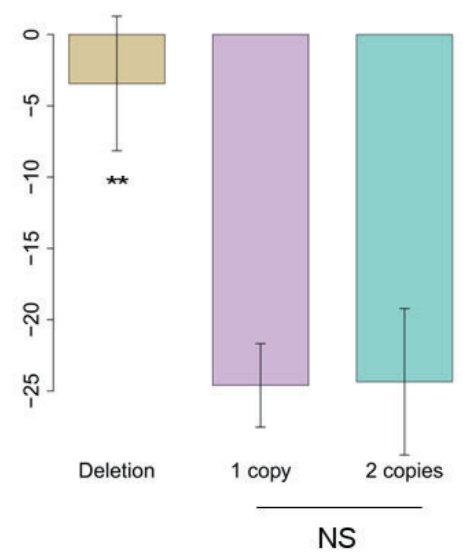

Worm genotype

Fig. 3. Inheritance of PZQ response in LE-PZQ population. Bar charts show the change in L-lactate production after exposure to $24 \mu \mathrm{g} / \mathrm{mL}$ PZQ in worms from different genotypic classes for QTL regions on chr. 2 and 3. (A). chr. 2 QTL (Kruskal-Wallis KW test $\left.\chi^{2}=0.019, \mathrm{p}=0.99\right)$, (B). Sm.TRPMPZQ-741987C $\left(\mathrm{KW}\right.$ test $\left.\chi^{2}=24.481, \mathrm{p}=2.93 \times 10^{-6}\right),(\mathbf{C}) .100 \mathrm{~kb}$ deletion $\left(\mathrm{KW}\right.$ test $\left.\chi^{2}=15.708, \mathrm{p}=0.0004\right)$. We see minimal change in L-lactate production following PZQ exposure in homozygotes for the SNP enriched in PZQ treated parasites, indicating that this trait is recessive. Parasites carrying two copies of the $100 \mathrm{~kb}$ deletion are also strongly associated with resistance, demonstrating that this deletion is in $\operatorname{LD}(N=120$ worms; NS: No significant difference between groups; $\left.{ }^{*} p<0.05 ; * * p \leq 0.01 ; * * * p \leq 0.001\right)$. 
the copy number variant and the SNP assayed in Sm.TRPMPZQ revealed that the causative gene in the chr. 3 QTL showed recessive inheritance. Homozygous parasites carrying two copies of the Sm.TRPMPZQ741987C allele (or two copies of the deletion) recovered from PZQ treatment, while the heterozygote and other homozygotes failed to recover from treatment (Fig. 3B-C). For the chr. 2 QTL, we did not see a significant association between parasite genotype and PZQ-R phenotype nor with lactate production before PZQ treatment (Fig. 3A): this locus was not investigated further.

\section{Marker-assisted purification of resistant and sensitive parasites}

As the chr. 3 QTL containing Sm.TRPMPZQ shows the strongest association with PZQ response and shows recessive inheritance, we were able to use single generation marker assisted selection approach to enrich parasites for alleles conferring PZQ resistance (PZQ-R) and PZQ sensitivity (PZQ-S) from the mixed genotype LE-PZQ-R parasite population (Fig. 4A). We genotyped cercariae larvae emerging from snails previously exposed to single miracidia to identify parasites homozygous for the recessive PZQ-R allele from those homozygous for the PZQ-S allele. Parasites isolated from multiple snails falling into these two alternative genotypes were then used to infect hamsters. The enriched PZQ-resistant and sensitive parasites were designated SmLE-PZQ-ER and SmLE-PZQ-ES. Sequencing of adult parasites recovered from these two populations revealed that they were fixed for alternative alleles at the Sm.TRPM $P Z Q^{-741987 C}$ SNP genotyped, but showed similar allele frequencies across the rest of the genome (Fig. S2). As expected, these sequences also revealed that the $100 \mathrm{~kb}$ deletion was close to fixation in the SmLE-PZQ-ER population (Fig. S3).

We conducted PZQ dose response curves on these enriched parasite populations. The SmLE-PZQ-ES population had an $\mathrm{IC}_{50}$ of $0.193 \mu \mathrm{g} / \mathrm{mL}$ ( \pm 1 s. d.: 0.045 ), while the SmLE-PZQ-ER population did not reach $50 \%$ reduction even at the highest dose $(72.9 \mu \mathrm{g} / \mathrm{mL})$ so has an $\mathrm{IC}_{50}>72.9 \mu \mathrm{g} / \mathrm{mL}$ : the two purified populations differ by $>377$-fold in PZQ response (Fig. 4B). These results provide further demonstration 
A.
Snails infected with LE-PZQ-R
Cercariae genotyping on chr.3 loci
Hamsters infection with cercariae $P Z Q-R$ or $P Z Q-S$

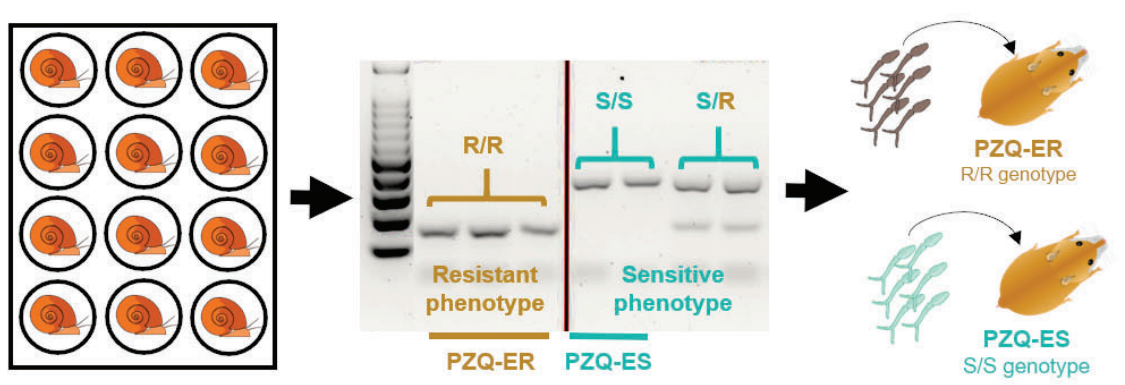

B.

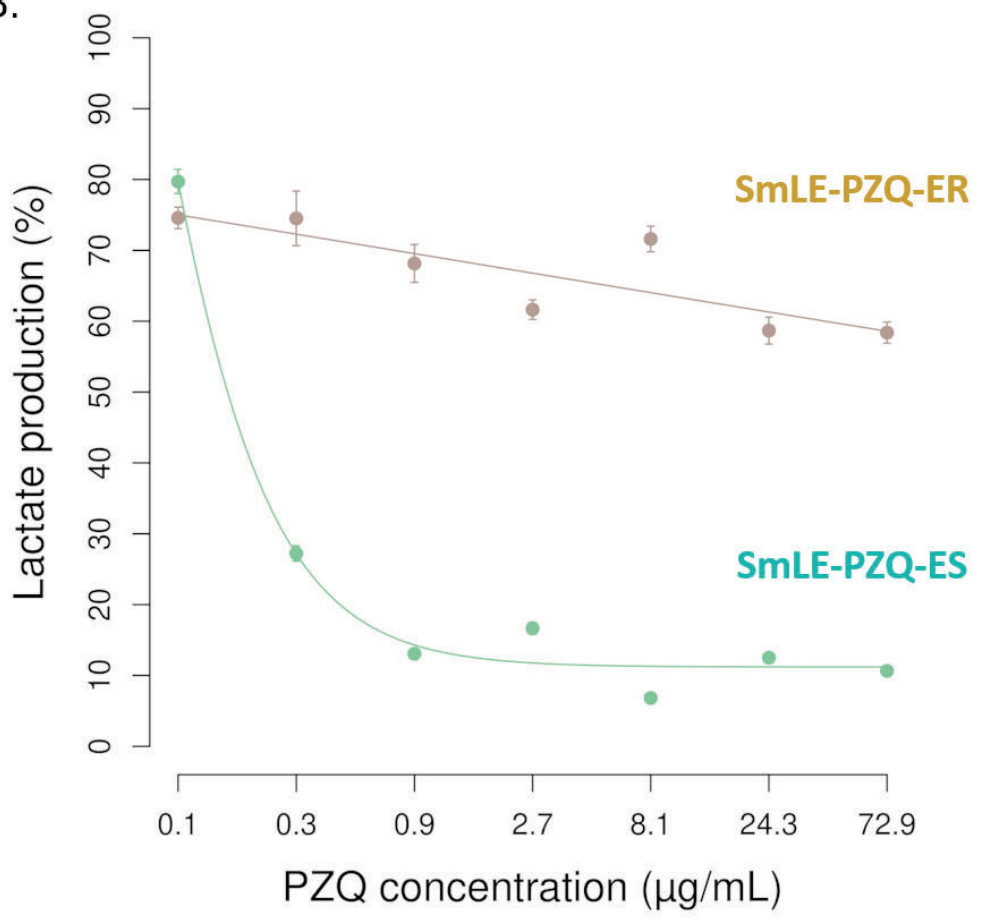

Fig. 4. Single generation marker-assisted purification of SmLE-PZQ-ES and SmLE-PZQ-ER parasites.

(A). Experimental strategy for identifying parasite larvae that are homozygous for Sm.TRPMPZQ alleles associated with PZQ-R or PZQ-S. We genotyped cercaria larvae emerging from snails infected with single parasite genotypes for a restriction site in the Sm.TRPMPZQ gene, and then infected two groups of hamsters with parasites homozygous for alternative alleles at this locus. (B). The two populations of parasites generated show dramatic differences in PZQ-response $\left(N=60\right.$ worms/population/treatment, $\chi^{2}$ test $\left.=373.03, \mathrm{p}<2.2 \times 10^{-16}\right)$. 
that the original LE-PZQ-R parasite population was a mixture of PZQ-R and PZQ-S parasites. Separation of the component SmLE-PZQ-ES and SmLE-PZQ-ER parasites from these mixed populations allows rigorous characterization of the PZQ-R trait in parasite populations that are fixed for alternative alleles at Sm.TRPM $P Z Q$, but contain comparable genomic backgrounds across the rest of the genome.

\section{Sm.TRPM $M_{P Z}$ gene and isoform expression is reduced in SmLE-PZQ-ER parasites}

PZQ response varies between parasite stages and sexes, with strongest response in adult males. Adult females and juvenile worms are naturally resistant $(31,32)$. We therefore examined gene expression in the purified SmLE-PZQ-ES and SmLE-PZQ-ER populations (males and females for both adults and juvenile worms) using RNA-seq (Fig. 5). Of the 85 genes expressed in adult worms under the chr. 3 QTL, only the Sm.TRPM $P Z Q$ showed a significant reduction in expression in the SmLE-PZQ-ER adult male worms relative to SmLE-PZQ-ES (2.25-fold, posterior probability =1) (Fig. 5A-B). Comparable under expression of Sm.TRPM $P Z Q$ was also seen in female when compared to SmLE-PZQ-ES: expression of Sm.TRPMPZQ was 11.94-fold lower in female than in male worms, consistent with females being naturally resistant $(31,32)$ (Fig. 5C). However, juvenile male and female worms showed elevated gene expression compared with adult worms (Fig. 5C). This is surprising because juveniles are naturally resistant to PZQ. Sm.TRPMPZQ has 41 exons and occurs as 7 isoforms containing between 3 and 36 exons. Strikingly, SmLE-PZQ-ES male worms showed a 4.02-fold higher expression of isoform 6 compared to SmLE-PZQ-ER males, and an 8fold higher expression than naturally resistant juvenile worms from both populations (while SmLE-PZQER showed only a 2-fold higher expression) (Fig. 5B and D and Fig. S4). This suggests that high expression of isoform 6 is linked to PZQ sensitivity. The 15 exons of isoform 6 produce an 836 amino acid protein that lacks the transmembrane domain but contains the TRPM domain. We interrogated the 10x single cell expression data from adult worms (46) showing that $S m . T R P M_{P Z Q}$ is expressed mainly in neural tissue with some expression also in muscle (Fig. S5). 
A.

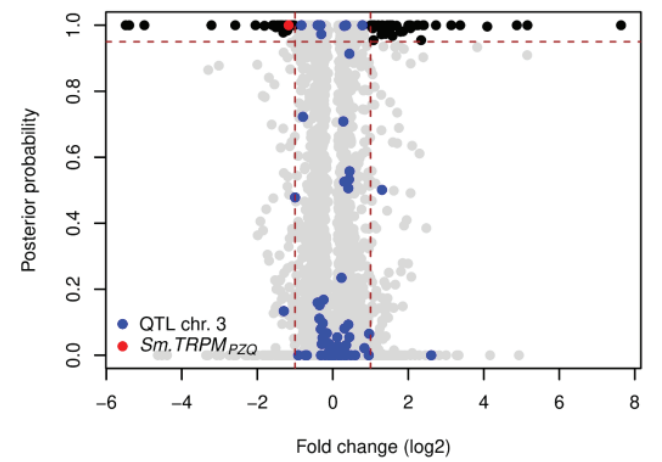

B. Isoform expression

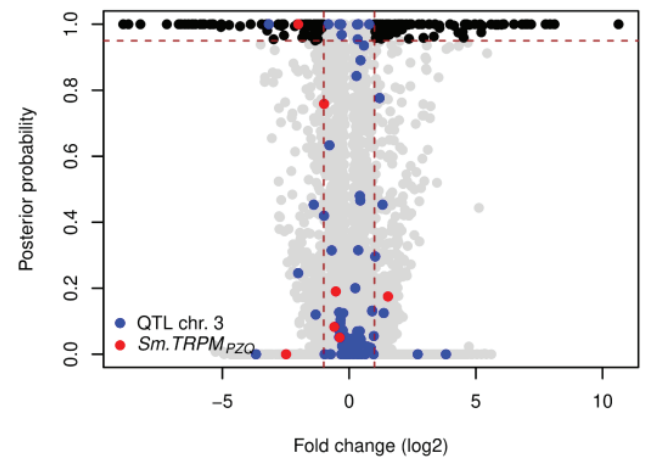

C. Sm.TRPM $P Z Q$ gene expression

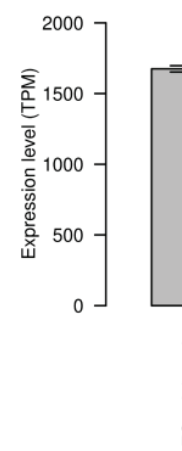

D.

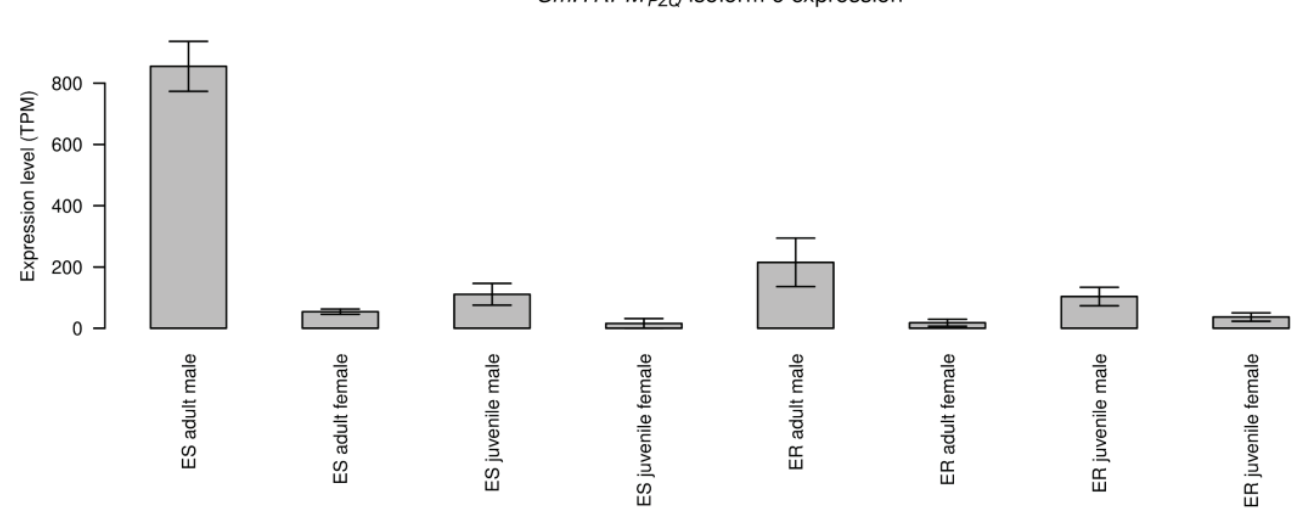

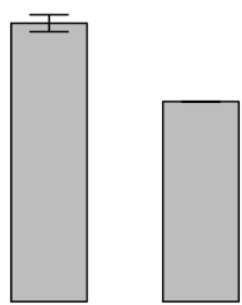

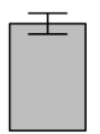

$\frac{0}{\pi}$
$\frac{\pi}{\tilde{z}}$
$\frac{5}{7}$
$\frac{\pi}{\pi}$
$\frac{\mathbb{4}}{4}$

Sm. TRPM $P Z Q$ isoform 6 expression
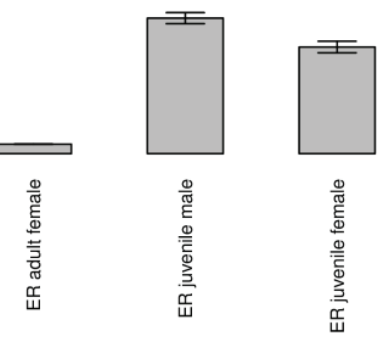

舀

Fig. 5. Gene expression differences between SmLE-PZQ-ES and SmLE-PZQ-ER parasites. Volcano plot showing the differential (A). gene expression and (B). isoform expression between adult male PZQ-ES and PZQ-ER (In blue: genes located under the chr. 3 QTL, in red: Sm.TRPMPZQ gene). (C). Sm.TRPMPZQ gene expression and (D). Sm.TRPMPZQ isoform 6 expression level comparison between PZQ-ES and ER for the two sex (i.e. male and females) and different stages (i.e. adult and juvenile). High expression of Sm.TRPMPZQ isoform 6 is linked to PZQ sensitivity. 
Both laboratory selected and field isolated S. mansoni showing PZQ-R have been difficult to maintain in the laboratory (47): the PZQ-R trait has been rapidly lost consistent with strong selection against this trait. It has been suggested that PZQ-R carries a fitness cost that will slow spread of this trait in the field under PZQ pressure. Such fitness costs are a common, but not ubiquitous, feature of drug resistance in other pathogens (48-50). We measured several components of parasite fitness in SmLE-PZQ-ES and SmLEPZQ-ER parasites during laboratory passage of purified parasite lines, but found no significant differences in infectivity to snails, snail survival, or infectivity to hamsters (Fig. 6A). We did not see loss of PZQ-R in our lines after 12 generations because the key genome region is fixed. Cioli et al. (28) has also reported

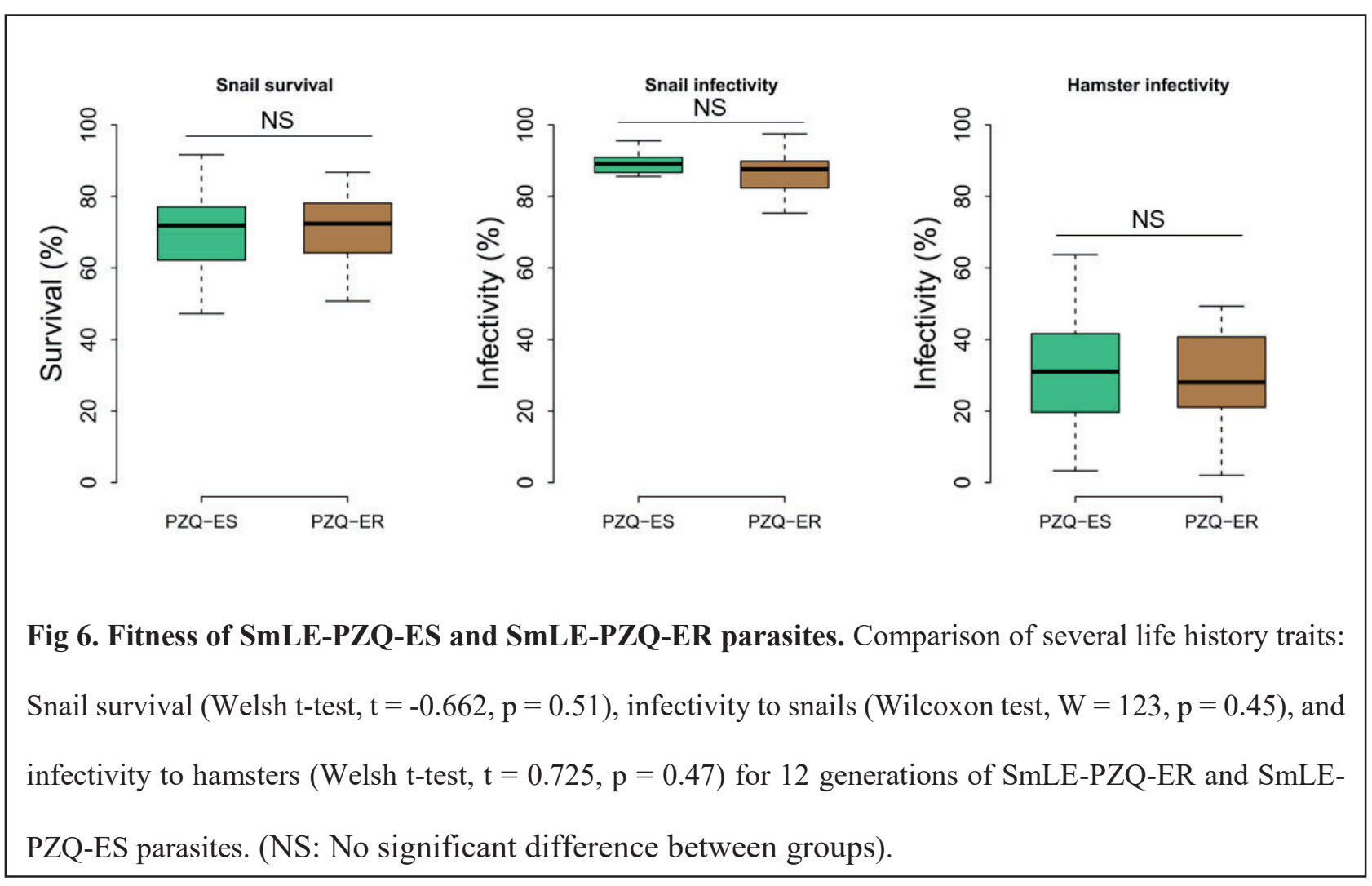


long term stability of PZQ-R parasite populations indicative that PZQ-R associated fitness costs maybe limited or absent.

\section{In vivo efficacy of PZQ against SmLE-PZQ-ES and SmLE-PZQ-ER parasites}

To determine the relationship between in vitro PZQ-R measured in 96-well plates, and in vivo resistance, we treated mice infected with either SmLE-PZQ-ER or SmLE-PZQ-ES parasites populations with $120 \mathrm{mg} / \mathrm{kg}$ of PZQ. We observed no significant reduction in worm burden in SmLE-PZQ-ER parasites when comparing PZQ-treated and control (DMSO) treated animals (Wilcoxon test, $p=0.393$; Fig. S6A). In contrast, we recovered significantly lower numbers of worms from PZQ-treated versus untreated mice infected with the SmLE-PZQ-ES parasite population (Wilcoxon test, $p=0.008$; Fig. S6A). The percent reduction observed was significantly different between the SmLE-PZQ-ES and SmLE-PZQ-ER parasites (Wilcoxon test, $\mathrm{p}=0.0129$; Fig. S6B). Interestingly, we observed a large reduction in numbers of female worms recovered from PZQ-treated SmLE-PZQ-ES parasites relative to untreated animals (Wilcoxon test, $p=0.008$; Fig. S6D), while for male worms this did not reach significance (Wilcoxon test, $p=0.089$, Fig. S6C). We saw no impact of PZQ-treatment for either female or male worms in mice infected with SmLEPZQ-ER. These results show that in vivo PZQ response in treated mice differs between SmLE-PZQ-ES and SmLE-PZQ-ER parasites. These data also suggest that the extended paralysis of male SmLE-PZQ-ES worms under PZQ treatment may reduce their ability to maintain female worms in copula.

\section{Chemical blockers and activators of Sm.TRPMPZQ modulate PZQ-R}

Sm.TRPM $P Z Q$ emerges as a strong candidate gene to explain variation in PZQ response, but validation is required. We were unsuccessful in knocking down expression of Sm.TRPMPZQ using either siRNA or dsRNA (Table S2), possibly because this gene is expressed mainly in neural tissue. We therefore used two 
chemical modulators of Sm.TRPMPZQ activity - an Sm.TRPMPZQ agonist (AG1) and Sm.TRPMPZQ antagonist (ANT1). These were identified from a screen of $\sim 16,000$ compounds by screening $\mathrm{Ca}^{2+}$ influx into HEK293 cells transiently expressing Sm.TRPMPZQ (Chulkov et al., in prep). Addition of the Sm.TRPMPZQ blocker (antagonist ANT1) allowed SmLE-PZQ-ES worms to recover from PZQ treatment (Fig. 7A), while the Sm.TRPMPZQ activator (agonist AG1) rendered SmLE-PZQ-ER worms sensitive to PZQ treatment in a dose dependent manner (Fig. 7B). These results are consistent with a role for Sm.TRPMPZQ in determining variation in PZQ response.

We found 5 non-synonymous SNPs and 5 insertions that showed significant differences in allele frequency in Sm.TRPMPZQ in the SmLE-PZQ-ES and SmLE-PZQ-ER parasite populations. One of these SNPs

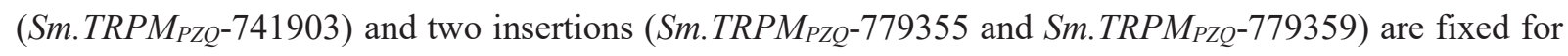
alternative alleles in the two populations, with 7 others are segregating at different frequencies in the two populations (Fig. S3). These SNPs are located outside the critical transmembrane domains so were not strong candidates to explain differences in PZQ-R. We expressed Sm.TRPMPZQ carrying some of these variants in HEK293 cells and examined their impact on $\mathrm{Ca}^{2+}$ influx to interrogate their role in explaining 

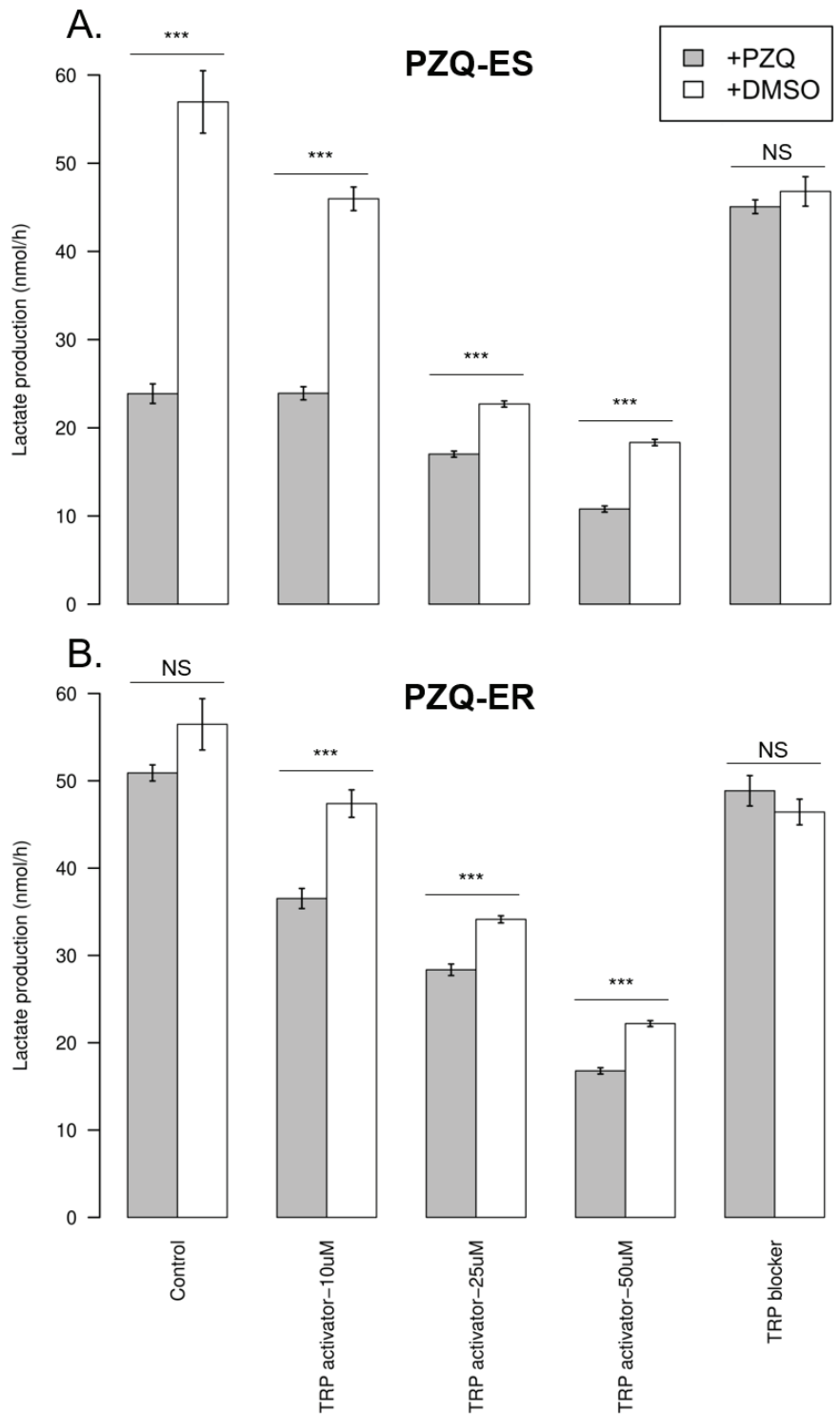

Fig. 7. Impact of Sm.TRPM $P Z Q$ blockers and activators on PZQ response. (A) SmLE-PZQ-ES and (B) SmLE-PZQER were exposed to either i) PZQ or DMSO alone (control group), ii) PZQ or DMSO combined with either $10 \mu \mathrm{M}$, $25 \mu \mathrm{M}$ or $50 \mu \mathrm{M}$ of Sm.TRPMPZQ activator (agonist AG1), iii) PZQ or DMSO combined with $50 \mu \mathrm{M}$ Sm.TRPMPZQ blocker (antagonist ANT1). Parasite viability was assessed 3 days post-treatment, based on their L-lactate production). Addition of the Sm.TRPMPZQ blocker allowed SmLE-PZQ-ES worms to recover from PZQ treatment (Welsh t-test, $\mathrm{t}$ $=-0.94, \mathrm{p}=0.35$ ), while the Sm.TRPMPZQ activator (agonist AG1) rendered SmLE-PZQ-ER worms sensitive to PZQ treatment in a dose dependent manner $(\mathrm{N}=20$ worms/population/treatment; Welsh t-test, NS: No significant difference between groups; ${ }^{*} p<0.05 ; * * p \leq 0.01 ; * * * p \leq 0.001$.) 
underlie PZQ response. We speculate that the difference in PZQ response is due to expression patterns and may be controlled by regulatory variants potentially associated with the adjacent $100 \mathrm{~kb}$ deletion or the SOX13 transcription factor.

\section{Sequence variation in $S m . T R P M_{P Z Q}$ from natural $S$. mansoni populations}

Methods for evaluating frequencies of PZQ-resistance mutations in endemic regions would provide a valuable tool for monitoring mass treatment programs aimed at schistosome elimination. Both this paper and the accompanying paper (21) identify $S m . T R P M_{P Z Q}$ as being critical to PZQ-response, and Park et al. have determined critical residues that determine binding between PZQ and Sm.TRPMPZQ (21). We examined the mutations present in $\operatorname{Sm} . T R P M_{P Z Q}$ in natural schistosome populations using exome

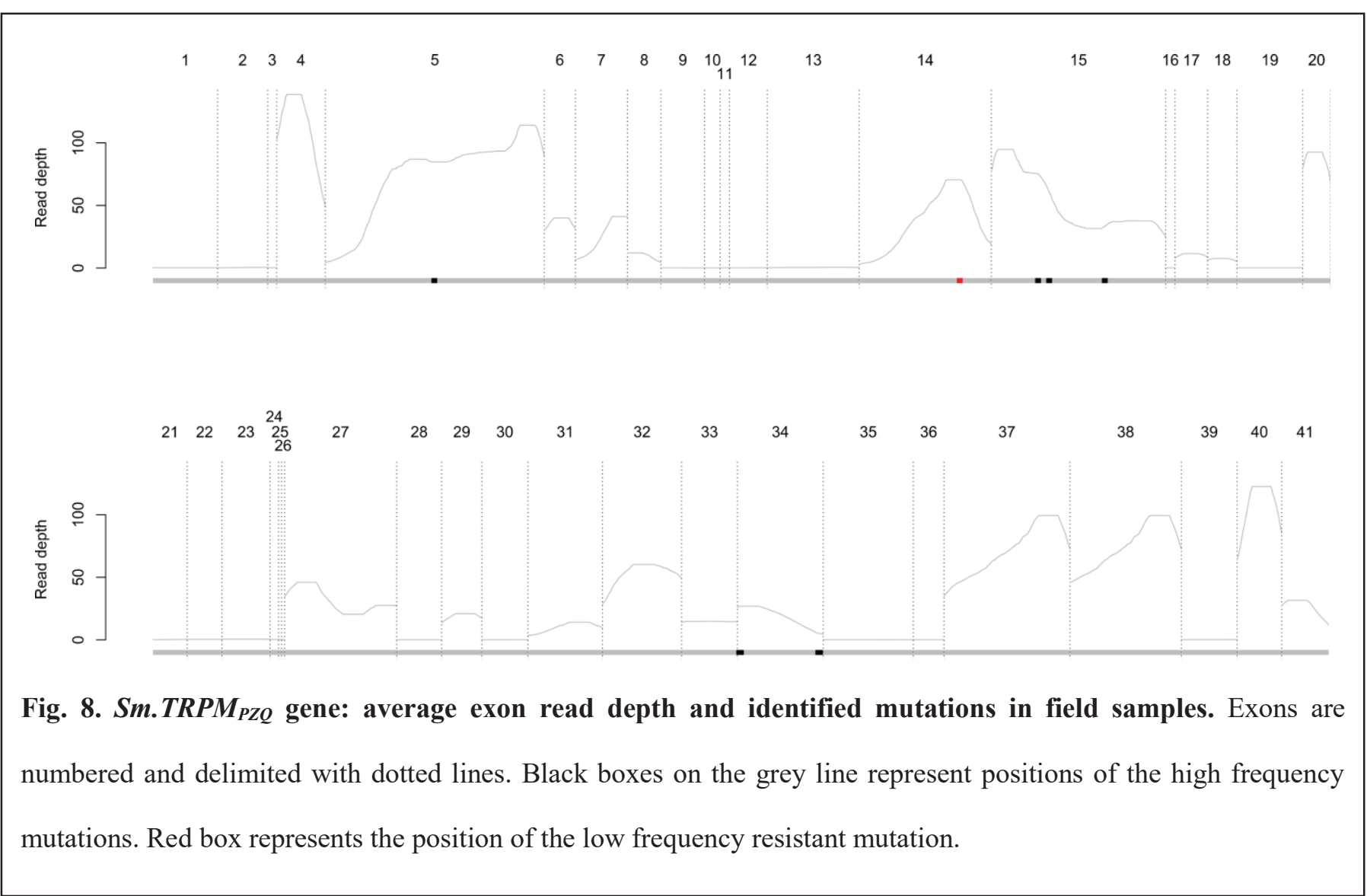


sequencing from 259 miracidia, cercariae or adult parasites from 3 African countries (Senegal, Niger, Tanzania), the Middle East (Oman) and South America (Brazil) $(51,52)$. We were able to sequence 36/41 exons of Sm.TRPMPZQ from 122/259 parasites on average (s.e. $=18.65)($ Table S3). We found 1 putative PZQ-R SNP in our Illumina reads supported by a very high coverage (Fig. S7). This SNP (c.2708G>T on isoform 5, p.G903*) was found in a single Omani sample and resulted in a truncated protein predicted to result in loss-of-function, demonstrating that PZQ-R alleles are present in natural populations. However, this PZQ-R allele observed was rare and present in heterozygous state so would not impact PZQ response (Fig. 8).

\section{DISCUSSION}

Our genetic approach to determining the genes underlying PZQ resistance - using GWAS and a simple lactate-based read out to determine parasite recovery following PZQ treatment in individual parasites robustly identifies a TRPM channel $\left(\operatorname{Sm} . T R P M_{P Z Q}\right)$ as the cause of variation in PZQ response. We were further able to purify SmLE-PZQ-ER and SmLE-PZQ-ES parasites to examine drug response and gene expression and to use chemical blockers to directly implicate $S m$.TRPM $P Z Q$. Our results complement those of Park et al. (21) who used a pharmacological approach to determine that Sm.TRPMPZQ is the major target for PZQ, and identified the critical residues necessary for activation by PZQ. Together, these approaches demonstrate that TRPM is a key determinant of schistosome response to PZQ.

A striking feature of the results is the strength of the PZQ-R phenotype. While previous authors have described quite modest differences (3-5 fold) in PZQ-response among $S$. mansoni isolates $(28,29)$, this study revealed at least 377 -fold difference in $\mathrm{IC}_{50}$ between SmLE-PZQ-ER and SmLE-PZQ-ES parasites. These large differences were only evident after we used marker-assisted selection to divide a mixed genotype laboratory S. mansoni population into component SmLE-PZQ-ER and SmLE-PZQ-ES populations. The modest $\mathrm{IC}_{50}$ differences in previous studies observed are most likely because the parasite 
lines compared contained mixed populations of both SmLE-PZQ-ER and SmLE-PZQ-ES individuals. This highlights a critical feature of laboratory schistosome populations that is frequently ignored: these populations are genetically variable and contain segregating genetic and phenotypic variation for a wide variety of parasite traits. In this respect they differ from the clonal bacterial or protozoan parasite "strains" used for laboratory research. Importantly, we can use this segregating genetic variation for genetic mapping of biomedically important parasite traits such as PZQ resistance.

There is strong evidence that PZQ-R parasites occur in schistosome populations in the field, but the contribution of PZQ-R to treatment failure in the field are unclear. Molecular markers are widely used for monitoring changes in drug resistance mutations in malaria parasites (53-55) and for evaluating benzimidazole resistance in nematode parasites of veterinary importance $(56,57)$. The discovery of the genetic basis of resistance to another schistosome drug (oxamniquine) (58) now makes genetic surveys possible to evaluate oxamniquine resistance in schistosome populations $(52,59)$. Identification of Sm.TRPM $P Z Q$ as a critical determinant of PZQ response, and determination of key residues that can underlie PZQ-R, now makes molecular surveillance possible for S. mansoni. We examined variation in Sm.TRPMPZQ in 259 parasites collected from locations from across the geographical range of this parasite. We were unable to confirm mutations in any of the key residues that block PZQ binding identified in the mutagenesis studies by Park et al. (21). However, we identified a stop codon in a single parasite isolated from a rodent from Oman (60) indicating a low frequency of PZQ-R resistance alleles $(1 / 502$, frequency $=0.002)$. This stop codon was in heterozygous state so is unlikely to result in PZQ-R.

These results are extremely encouraging for control programs, but should be viewed with considerable caution for two reasons. First, we do not know yet the regulatory regions of $S m . T R P M_{P Z Q}$ and we were unable to identify regulatory variants of $S m . T R P M_{P Z Q}$ in this screen. Such variants could reduce expression of Sm.TRPM $P Z Q$ resulting in PZQ resistance. We note that coding variants underlying PZQ-R phenotype were not found in our laboratory SmLE-PZQ-ER parasites, suggesting that regulatory changes may underlie this trait. Second, Sm.TRPM $P Z Q$ is a large gene (120 kb and 41 exons) that is poorly captured by genome 
sequencing of field samples. We were able to successfully sequence $36 / 41$ exons, including those that directly interact with PZQ (21), using exome capture methods (51, 52). However, improved sequence coverage will be needed for full length sequencing of this gene. Third, the parasite samples we examined did not come from hotspot regions where regular mass drug administration of PZQ has failed to reduce $S$. mansoni burdens $(36,61)$. Targeted sequencing of miracidia from these populations will be extremely valuable to determine if there are local elevations in Sm.TRPMPZQ variants, or if particular variants are enriched in parasites surviving PZQ treatment. Ideally, such sequence surveys should be partnered with functional validation studies in which variant $S m . T R P M_{P Z Q}$ are expressed in HEK293 cells to determine their response to PZQ exposure (21).

\section{MATERIALS AND METHODS}

\section{Study design}

This study was designed to determine the genetic basis of PZQ-R, and was stimulated by the initial observation that a laboratory $S$. mansoni population generated through selection with PZQ contained both PZQ-S and PZQ-R individuals. The project had 6 stages:(i) QTL location. We conducted a genome-wide association study (GWAS). This involved measuring the PZQ-response of individual worms, pooling those showing high levels of resistance and low levels of resistance, sequencing the pools to high read depth, and then identifying the genome regions showing significant differences in allele frequencies between high and low resistance parasites. (ii) Fine mapping of candidate genes. We identified potential candidate genes in these QTL regions, through examination of gene annotations, and exclusion of genes that are not expressed in adults. We also determined whether the loci determining PZQ-R are inherited in a recessive, dominant or co-dominant manner (iii) Marker assisted purification of PZQ-S and PZQ-R parasites. To separate PZQ$\mathrm{R}$ and PZQ-S parasites into "pure" populations, we genotyped larval parasites for genetic markers in the QTL regions and infected rodents with genotypes associated with PZQ-R or PZQ-S. To verify that this 
approach worked, we then measured the $\mathrm{IC}_{50}$ for each of the purified populations. (iv) Characterization of purified SmLE-PZQ-ER and SmLE-PZQ-ES populations. Separation of SmLE-PZQ-ES and SmLE-PZQER parasite populations allowed us to characterize these in more detail. Specifically, we measured expression in juvenile and adult worms of both sexes in both SmLE-PZQ-ES and SmLE-PZQ-ER parasites. We also quantified parasite fitness traits. (v) Functional analysis. We used RNAi and chemical manipulation approaches to modulate activity of candidate genes and determine the impact of PZQ-resistance. We also used transient expression of candidate genes in cultured mammalian cells, to determine the impact of particular SNPs on response to PZQ-exposure. (vi) Survey of PZQ-resistance variants in field collected parasites. Having determined the gene underlying PZQ-R in laboratory parasites, we examined sequence variation in this gene in a field collection of $S$. mansoni parasites collected to examine the frequency of sequence variants predicted to result in PZQ-resistance. Methods are described in detail (File S1) and in brief below.

\section{$\underline{\text { Ethics statement }}$}

This study was performed following the Guide for the Care and Use of Laboratory Animals of the National Institutes of Health. The protocol was approved by the Institutional Animal Care and Use Committee of Texas Biomedical Research Institute (permit number: 1419-MA and 1420-MU). Ethical permission for collection of samples from humans are described in $(51,52)$.

\section{Biomphalaria glabrata snails and Schistosoma mansoni parasites}

We used uninfected inbred 8 - 10 mm albino Biomphalaria glabrata snails (line Bg121 (62)). The SmLE S. mansoni population was originally obtained from an infected patient in Brazil (63). The SmLE-PZQ-R schistosome population was generated by applying a single round of PZQ selection pressure on SmLE parasites at both snail and rodent stages (26) and has been maintained in our laboratory since 2014 .

\section{Drug resistance tests:}


Dose-response curves to PZQ in SmLE and SmLE-PZQ-R populations

We initially measured PZQ sensitivity by examining worm motility (64) in SmLE and SmLE-PZQ-R parasite populations. Ten adult males from SmLE or SmLE-PZQ-R populations were placed into each well of a 24-well microplate containing $1 \mathrm{~mL}$ DMEM complete media (65). We performed control and experimental groups in triplicate ( $N=240$ worms/parasite population). We exposed adult worms to PZQ $(0$, $0.10 .3,0.9,2.7,8.1,24.3$ and $72.9 \mu \mathrm{g} / \mathrm{mL}$ ) for $24 \mathrm{~h}$. Worms were washed (3x) in drug-free medium and incubated $\left(37^{\circ} \mathrm{C}, 5 \% \mathrm{CO}_{2}\right)$ for 2 days. The parasites were observed daily for the 5 days and the number of dead worms scored. Worms were defined as "dead" if they showed no movements and became opaque.

\section{Metabolic assessment of worm viability using L-lactate assay}

We adapted a method for metabolic assessment of worm viability using an L-lactate assay (44). Adult male SmLE-PZQ-R worms were placed individually in 96-well plates containing $100 \mu \mathrm{m}$ mesh filter insert (Millipore) in $250 \mu \mathrm{L}$ DMEM complete media. We added PZQ $(24.3 \mu \mathrm{g} / \mathrm{mL}$ in DMEM complete media) while controls were treated with the same volume of drug diluent DMSO. At $48 \mathrm{~h}$ post-treatment, the supernatant $(125 \mu \mathrm{L})$ was collected from each well and immediately stored at $-80{ }^{\circ} \mathrm{C}$ until processing. $\mathrm{We}$ measured lactate levels in the supernatants of in vitro treated adult male worms with a colorimetric L-lactate assay kit (Sigma) using 96-well, optical clear-bottom plates (Corning).

\section{Genome wide association analysis and QTL mapping}

$\underline{\text { Schistosome infections }}$

We collected eggs from livers of hamsters infected with SmLE-PZQ-R (66) and exposed one thousand Bg121 snails to five miracidia/snail. After 30 days, snails were individually exposed to light to shed cercariae. We exposed eight hamsters to 840 cercariae (4 cercariae/snail) from a batch of 210 shedding snails. We euthanized hamsters after 45 days to collect adult worms.

\section{Phenotypic selection}


We plated adult SmLE-PZQ-R males individually in 96 -well plates (60 worms per plate) in $250 \mu \mathrm{L}$ of DMEM complete media and treated with a $24.3 \mu \mathrm{g} / \mathrm{mL}$ PZQ. A group of 12 worms was treated with the same volume of drug diluent DMSO. This GWAS experiment was done twice independently. A total of 590 and 691 adult male worms were collected, cultured in vitro and exposed to PZQ for the two experiments.

We collected media supernatants $(125 \mu \mathrm{L})$ in $96-$ well PCR plates after $24 \mathrm{~h}$ in culture (pre-treatment) and $48 \mathrm{~h}$ post-treatment for quantifying lactate levels. We took the $20 \%$ of the treated worms releasing the highest amount of lactate (average L-lactate production \pm SD: Experiment $1=61.44 \mathrm{nmol} / \mathrm{h} \pm 13.16$ / Experiment $2=56.38 \mathrm{nmol} / \mathrm{h} \pm 10.82$ ) and the $20 \%$ of the treated worms releasing the lowest amount of lactate (average L-lactate production $\pm \mathrm{SD}$ : Experiment $1=28.61 \mathrm{nmol} / \mathrm{h} \pm 5.32 /$ Experiment $2=23.04$ $\mathrm{nmol} / \mathrm{h} \pm 4.14), 48 \mathrm{~h}$ post PZQ treatment.

\section{DNA extraction and library preparation}

We sequenced whole genomes of the two pools of recovered (Experiment 1: 116 worms / Experiment 2: 137 worms) and susceptible worms (Experiment 1: 116 worms / Experiment 2: 137 worms) and measured allele frequencies in each pool to identify genome regions showing high differentiation. We extracted gDNA from pools of worms (Blood and Tissue kit, Qiagen) and prepared whole genome libraries in triplicate (KAPA HyperPlus kit, KAPA Biosystems). Raw sequence data are available at the NCBI Sequence Read Archive (PRJNA699326).

\section{$\underline{\text { Bioinformatic analysis }}$}

We used Jupyter notebook and scripts used for processing the sequencing data and identifying the QTL (https://github.com/fdchevalier/PZQ-R_DNA).

\section{a. Sequence analysis and variant calling}

We (i) aligned the sequencing data against the S. mansoni reference genome using BWA (67) and SAMtools (68), (ii) used GATK $(69,70)$ to mark PCR duplicates and recalibrate base scores, (iii) used the 
HaplotypeCaller module of GATK to call variants (SNP/indel) and GenotypeGVCFs module to perform joint genotyping. We merged VCF files using the MergeVcfs module. All steps were automated using Snakemake (71).

\section{b. QTL identification}

We examined the difference in allele frequencies between low and high lactate parasites across the genome by calculating a $Z$-score at each bi-allelic site. We weighed $Z$-scores by including the number of worms in each treatment and the difference in the total read depth across the triplicated libraries of each treatment at the given variant. We combined $Z$-scores generated from each biological replicate. Bonferroni correction was calculated with $\alpha=0.05$.

\section{$\underline{\text { Relationship between worm genotype at chr. } 2 \text { and } 3 \text { and PZQ-R phenotype }}$}

To validate the impact of worm genotypes on PZQ resistance phenotype and to determine whether PZQ-R shows recessive, dominant or codominant inheritance, we measured the PZQ-R phenotype of individual worms, and genotyped worms for markers at the peak of the QTLs located.

\section{Measuring $P Z Q-R$ in individual worms}

We plated 120 SmLE-PZQ-R adult male worms individually in 96-well plates, treated them with PZQ (24.3 $\mu \mathrm{g} / \mathrm{mL})$ and collected media supernatants pre- $(24 \mathrm{~h})$ and post- $(48 \mathrm{~h})$ treatment, and used L-lactate assays to determine PZQ-R status. We extracted gDNA from each worm individually (66).

\section{PCR-RFLP for chr. 2 and chr. 3 loci}

We used PCR-RFLP to genotype single worms at loci marking QTL peaks on chr. 2 (C>A, chr SM_V7_2: 1072148) and chr. 3 (T>C, chr SM_V7_3: 741987) (Table S4). We digested PCR amplicons for chr. 2 with BslI (NEB) and chr. 3 with Mse1 (NEB), and visualized digested PCR amplicons by 2\% agarose gel electrophoresis. 
Quantitative PCR of copy number variation (CNV) in single worms

We genotyped individual worms for a deletion on chr. 3 (position 1220683-1220861 bp) using a qPCR assay. Methods and primers are described in Table S4.

\section{$\underline{\text { Marker assisted selection of resistant and susceptible parasite populations }}$}

\section{Selection of SmLE-PZQ-ER and SmLE-PZQ-ES populations}

We separated the polymorphic SmLE-PZQ-R schistosome population based on chr. 3 QTL genotype using PCR-RFLP. We exposed 960 inbred B. glabrata Bg121 snails to one miracidium SmLE-PZQ-R (66). At four weeks post-exposure, we identified infected snails $(N=272)$, collected cercariae from individual snails, extracted cercarial DNA (66)), and genotyped each parasite for the chr. 3 locus using PCR-RFLP (Homozygous R/R: $n=89-36 \%$; Homozygous $S / S$ : $n=39-16 \%$; Heterozygous R/S: $n=117-49 \%$ ), and determined their gender by PCR (72). We selected $32 \mathrm{R} / \mathrm{R}$ parasites and $32 \mathrm{~S} / \mathrm{S}$ genotypes. For both R/R and $\mathrm{S} / \mathrm{S}$ we used 13 males and 19 females. We exposed 5 hamsters to 800 cercariae of $32 \mathrm{R} / \mathrm{R}$ genotypes parasites and 5 hamsters to 800 cercariae $32 \mathrm{~S} / \mathrm{S}$ genotyped parasites. This single generation marker assisted selection procedure generates two subpopulations: SmLE-PZQ-ER is enriched for parasites with $R / R$ genotype, while SmLE-PZQ-ES is enriched for S/S genotypes.

\section{$\underline{P Z Q I C_{50}}$ with SmLE-PZQ-ER and SmLE-PZQ-ES}

Forty-five days after infection, we euthanized and perfused hamsters to recover adult schistosome worms for SmLE-PZQ-ER and SmLE-PZQ-ES subpopulations. We placed adult males in 96-well plates and cultured in $250 \mu \mathrm{L}$ DMEM complete media. We determined PZQ dose-response for both SmLE-PZQ-ER and SmLE-PZQ-ES population.

gDNA extraction and library preparation and bioinformatics

We recovered the F1 SmLE-PZQ-ER and SmLE-PZQ-ES worms and extracted gDNA from pools of adult males or females separately. We prepared whole genome libraries from these pools in triplicate using the 
KAPA HyperPlus kit (KAPA Biosystems). Sequence data are available at the NCBI Sequence Read Archive (accession numbers PRJNA701978). The analysis was identical to the GWAS and QTL mapping analysis (see Jupyter notebook and scripts (https://github.com/fdchevalier/PZQ-R_DNA)).

\section{Transcriptomic analysis of resistant and susceptible schistosome worms at juvenile and adult stages}

\section{Sample collection}

We recovered S. mansoni SmLE-PZQ-ER and SmLE-PZQ-ES worms by perfusion from hamsters at 28 days (juveniles) or 45 days (adults) post-infection. For each subpopulation, (SmLE-PZQ-ER or SmLEPZQ-ES), we collected 3 biological replicates of 30 males or 30 females for the $28 \mathrm{~d}$ juvenile worms, and 3 biological replicates of 30 males or 60 females for the $45 \mathrm{~d}$ adult worms.

\section{RNA extraction and RNAseq library preparation}

\section{a. RNA extraction}

We extracted total RNA from all the S. mansoni adult and juvenile worms (RNeasy Mini kit, Qiagen), quantified the RNA recovered (Qubit RNA Assay Kit, Invitrogen) and assessed the RNA integrity by TapeStation (Agilent - RNA integrity numbers: $\sim 8.5-10$ for all samples).

\section{b. RNAseq library preparation}

We prepared RNAseq libraries using the KAPA Stranded mRNA-seq kit (KAPA Biosystems) using 500ng RNA for each library. We sequenced the libraries using $150 \mathrm{bp}$ pair-end reads. Raw sequence data are available at the NCBI Sequence Read Archive (accession numbers PRJNA704646).

\section{c. Bioinformatic analysis.}

To identify differentially expressed genes, we aligned the sequencing data against the $S$. mansoni reference genome using STAR. We quantified gene and isoform abundances by computing transcripts per million values using RSEM and compared abundances between groups (ES/ER, males/females, juveniles/adults) using the R package EBSeq. 


\section{Manipulation of $S m . T R P M P Z O$ channel expression or function:}

\section{$\underline{R N A \text { interference }}$}

We attempted RNA interference to functionally validate the implication of Sm.TRPMPZQ on schistosome PZQ resistance. The methods used are described in File S1 and Table S2, but were unsuccessful so are not described here.

Specific Sm.TRPMPZO chemical inhibitor and activators

We used specific chemical inhibitor and activators (Chulkov et al., in prep) to manipulate the function of

Sm.TRPMPZQ to examine the impact on PZQ-response. We placed individual SmLE-PZQ-ER and SmLE-

PZQ-ES adult male worms in 96-well plates containing DMEM complete media. After 24h, 20 worms from

each population were treated either with a cocktail combining PZQ $(1 \mu \mathrm{g} / \mathrm{mL})$ and i) $50 \mu \mathrm{M}$ of Sm.TRPMPZQ

blocker (MB2) or ii) $10 \mu \mathrm{M}, 25 \mu \mathrm{M}$ or $50 \mu \mathrm{M}$ of $S m$.TRPMPZQ activator (MV1) respectively or iii) drug incubated $\left(37^{\circ} \mathrm{C}, 5 \% \mathrm{CO}_{2}\right)$ for 2 days. We collected media supernatants $(125 \mu \mathrm{L})$ before treatment $($ after $24 \mathrm{~h}$ in culture) and $48 \mathrm{~h}$ post-treatment and quantified lactate levels.

\section{$\underline{\text { In vivo parasite survival after } P Z Q \text { treatment }}$}

We split 24 female Balb/C mice into two groups: one group exposed by tail immersion to SmLE-PZQ-ER (80 cercariae/mouse from 40 infected snails) and the second one to SmLE-PZQ-ES (80 cercariae/mouse of PZQ (1\% DMSO + vegetable oil in a total volume of $150 \mu \mathrm{L})$ or the same volume of drug diluent only

\section{$\underline{\text { Sm.TRPM }}$ PZO variants in S. mansoni field samples}


518 We utilized exome sequence data from S. mansoni from Africa, South America and the middle East to investigate variation in Sm.TRPMPZQ. African miracidia were from the Schistosomiasis collection at the Natural History Museum (SCAN) (73), Brazilian miracidia and Omani cercariae and adult worms were collected previously. We have previously described exome sequencing methods for $S$. mansoni $(51,52)$. Data were analyzed the same way as described in Chevalier et al. (52). Code is available in Jupyter notebook and scripts associated (https://github.com/fdchevalier/PZQ-R_field).

Sanger re-sequencing to confirm the presence of the $\mathrm{Sm}_{\text {TRPMPZQ field variants }}$

To confirm the presence of variants in Sm.TRPMPZQ (when read depth was $<10$ reads), we performed Sanger re-sequencing of mutations of interest close to the PZQ binding site (21). Primers and conditions are listed in Table S4. We scored variants using PolyPhred software (v6.18) (Nickerson et al., 1997). Sequences are deposited in GenBank (KU951903-KU952091).

\section{$\underline{\text { Statistical analysis }}$}

Statistical analyzes and graphs were performed using R software (v3.5.1) (74). We used the drc package from R to analyze dose-response datasets and Readqpcr and Normqper packages to analyze RT-qPCR $95 \%$ and $p$-values $<0.05$ were considered significant.

\section{Supplementary Materials}

Fig. S1. Development of a lactate assay for assaying worm recovery. 

available under aCC-BY-NC 4.0 International license.

Fig. S2. Validation of marker-assisted selection of SmLE-PZQ-ER and ES using Next Sequencing (NGS).

Fig. S3. Large deletions adjacent to Sm.TRPMPZQ and SOX13 transcription factor.

Fig. S4. Detailed genes and isoforms expression in SmLE-PZQ-ER and SmLE-PZQ-ES parasites.

Fig. S5. Cellular localization of Sm.TRPMPZQ expression in S. mansoni.

Fig. S6. Impact of in vivo PZQ treatment on SmLE-PZQ-ER and SmLE-PZQ-ES parasites.

Fig. S7. Stop codon identified in S. mansoni field sample from Oman.

Table S1. Genes in QTL regions on chr. 2 and 3.

Table S2. Details of RNAi for Sm.TRPMPZQ.

Table S3. Mutations present in Sm.TRPMPZQ in natural schistosome populations from 3 African countries (Senegal, Niger, Tanzania), the Middle East (Oman) and South America (Brazil).

Table S4. Summary table of siRNA sequences and primer sequences used for PCR-RFLP, RT-qPCR, PCRs and Sanger sequencing.

File S1. Expanded Material and Methods. 


\section{References and Notes}

1. World Health Organization, Schistosomiasis, (available at https://www.who.int/news-room/factsheets/detail/schistosomiasis).

2. G. B. D. 2017 Disease, I. Incidence, P. Collaborators, Global, regional, and national incidence, prevalence, and years lived with disability for 354 diseases and injuries for 195 countries and territories, 1990-2017: a systematic analysis for the Global Burden of Disease Study 2017., Lancet (London, England) 392, 1789-1858 (2018).

3. D. P. McManus, D. W. Dunne, M. Sacko, J. Utzinger, B. J. Vennervald, X.-N. Zhou, Schistosomiasis., Nature reviews. Disease primers 4, 13 (2018).

4. G. B. D. 2015 Mortality, C. of Death Collaborators, Global, regional, and national life expectancy, all-cause mortality, and cause-specific mortality for 249 causes of death, 1980-2015: a systematic analysis for the Global Burden of Disease Study 2015., Lancet (London, England) 388, 1459-1544 (2016).

5. M. A. Verjee, Schistosomiasis: Still a cause of significant morbidity and mortality., Research and reports in tropical medicine 10, 153-163 (2019).

6. M. J. van der Werf, S. J. de Vlas, S. Brooker, C. W. N. Looman, N. J. D. Nagelkerke, J. D. F. Habbema, D. Engels, Quantification of clinical morbidity associated with schistosome infection in sub-Saharan Africa., Acta Trop 86, 125-139 (2003).

7. W. H. Organization, others, Schistosomiasis: progress report 2001-2011, strategic plan 2012-2020, (2013).

8. A. K. Deol, F. M. Fleming, B. Calvo-Urbano, M. Walker, V. Bucumi, I. Gnandou, E. M. Tukahebwa, S. Jemu, U. J. Mwingira, A. Alkohlani, M. Traoré, E. Ruberanziza, S. Touré, M.-G. Basáñez, M. D. French, J. P. Webster, Schistosomiasis - assessing progress toward the 2020 and 2025 global goals., The New England journal of medicine 381, 2519-2528 (2019).

9. S. H. Xiao, P. A. Friedman, B. A. Catto, L. T. Webster, Praziquantel-induced vesicle formation in the tegument of male Schistosoma mansoni is calcium dependent., The Journal of parasitology 70, 177-179 (1984).

10. R. Pax, J. L. Bennett, R. Fetterer, A benzodiazepine derivative and praziquantel: effects on musculature of Schistosoma mansoni and Schistosoma japonicum., Naunyn-Schmiedeberg's archives of pharmacology 304, 309315 (1978).

11. B. Becker, H. Mehlhorn, P. Andrews, H. Thomas, J. Eckert, Light and electron microscopic studies on the effect of praziquantel on Schistosoma mansoni, Dicrocoelium dendriticum, and Fasciola hepatica (Trematoda) in vitro., Zeitschrift fur Parasitenkunde (Berlin, Germany) 63, 113-128 (1980).

12. C. S. Bricker, J. W. Depenbusch, J. L. Bennett, D. P. Thompson, The relationship between tegumental disruption and muscle contraction in Schistosoma mansoni exposed to various compounds., Zeitschrift fur Parasitenkunde (Berlin, Germany) 69, 61-71 (1983).

13. P. M. Cupit, C. Cunningham, What is the mechanism of action of praziquantel and how might resistance strike?, Future medicinal chemistry 7, 701-705 (2015).

14. C. M. Thomas, D. J. Timson, The mechanism of action of praziquantel: Six hypotheses., Current topics in medicinal chemistry 18, 1575-1584 (2018).

15. A. B. Kohn, P. A. Anderson, J. M. Roberts-Misterly, R. M. Greenberg, Schistosome calcium channel beta subunits. Unusual modulatory effects and potential role in the action of the antischistosomal drug praziquantel., The Journal of biological chemistry 276, 36873-36876 (2001). 

available under aCC-BY-NC 4.0 International license.

16. M. C. Jeziorski, R. M. Greenberg, Voltage-gated calcium channel subunits from platyhelminths: potential role in praziquantel action., International journal for parasitology 36, 625-632 (2006).

17. J. D. Chan, M. Zarowiecki, J. S. Marchant, $\mathrm{Ca}^{2+}$ channels and praziquantel: a view from the free world., Parasitology international 62, 619-628 (2013).

18. R. S. Kasinathan, L. K. Sharma, C. Cunningham, T. R. Webb, R. M. Greenberg, Inhibition or knockdown of $\mathrm{ABC}$ transporters enhances susceptibility of adult and juvenile schistosomes to Praziquantel., PLoS neglected tropical diseases $\mathbf{8}$, e3265 (2014).

19. R. M. Greenberg, ABC multidrug transporters in schistosomes and other parasitic flatworms., Parasitology international 62, 647-653 (2013).

20. S.-K. Park, G. S. Gunaratne, E. G. Chulkov, F. Moehring, P. McCusker, P. I. Dosa, J. D. Chan, C. L. Stucky, J. S. Marchant, The anthelmintic drug praziquantel activates a schistosome transient receptor potential channel., The Journal of biological chemistry 294, 18873-18880 (2019).

21. S.-K. Park, L. Friedrich, N. A. Yahya, C. Rohr, E. G. Chulkov, D. Maillard, F. Rippmann, T. Spangenberg, J. S. Marchant, Mechanism of praziquantel action at a parasitic flatworm ion channel, bioRxiv (2021), doi:10.1101/2021.03.09.434291.

22. P. G. Fallon, M. J. Doenhoff, Drug-resistant schistosomiasis: resistance to praziquantel and oxamniquine induced in Schistosoma mansoni in mice is drug specific., Am J Trop Med Hyg 51, 83-88 (1994).

23. B. Gryseels, L. Nkulikyinka, D. Engels, Impact of repeated community-based selective chemotherapy on morbidity due to schistosomiasis mansoni., The American journal of tropical medicine and hygiene 51, 634-641 (1994).

24. M. Ismail, S. Botros, A. Metwally, S. William, A. Farghally, L. F. Tao, T. A. Day, J. L. Bennett, Resistance to praziquantel: direct evidence from Schistosoma mansoni isolated from Egyptian villagers., The American journal of tropical medicine and hygiene 60, 932-935 (1999).

25. D. Alonso, J. Muñoz, J. Gascón, M. E. Valls, M. Corachan, Failure of standard treatment with praziquantel in two returned travelers with Schistosoma haematobium infection., The American journal of tropical medicine and hygiene 74, 342-344 (2006).

26. F. F. B. Couto, P. M. Z. Coelho, N. Araújo, J. R. Kusel, N. Katz, L. K. Jannotti-Passos, A. C. A. Mattos, Schistosoma mansoni: a method for inducing resistance to praziquantel using infected Biomphalaria glabrata snails., Memorias do Instituto Oswaldo Cruz 106, 153-157 (2011).

27. W. Wang, L. Wang, Y.-S. Liang, Susceptibility or resistance of praziquantel in human schistosomiasis: a review., Parasitology research 111, 1871-1877 (2012).

28. D. Cioli, S. S. Botros, K. Wheatcroft-Francklow, A. Mbaye, V. Southgate, L.-A. T. Tchuenté, L. Pica-Mattoccia, A. R. Troiani, S. H. S. El-Din, A.-N. A. Sabra, J. Albin, D. Engels, M. J. Doenhoff, Determination of ED50 values for praziquantel in praziquantel-resistant and -susceptible Schistosoma mansoni isolates., International journal for parasitology 34, 979-987 (2004).

29. L. Pica-Mattoccia, M. J. Doenhoff, C. Valle, A. Basso, A.-R. Troiani, P. Liberti, A. Festucci, A. Guidi, D. Cioli, Genetic analysis of decreased praziquantel sensitivity in a laboratory strain of Schistosoma mansoni., Acta Trop 111, 82-85 (2009).

30. J. Zwang, P. Olliaro, Efficacy and safety of praziquantel $40 \mathrm{mg} / \mathrm{kg}$ in preschool-aged and school-aged children: a meta-analysis., Parasites \& vectors 10, 47 (2017). 

available under aCC-BY-NC 4.0 International license.

31. L. Pica-Mattoccia, D. Cioli, Sex- and stage-related sensitivity of Schistosoma mansoni to in vivo and in vitro praziquantel treatment., International journal for parasitology 34, 527-533 (2004).

32. S. Botros, L. Pica-Mattoccia, S. William, N. El-Lakkani, D. Cioli, Effect of praziquantel on the immature stages of Schistosoma haematobium., International journal for parasitology 35, 1453-1457 (2005).

33. M. Ismail, A. Metwally, A. Farghaly, J. Bruce, L. F. Tao, J. L. Bennett, Characterization of isolates of Schistosoma mansoni from Egyptian villagers that tolerate high doses of praziquantel., The American journal of tropical medicine and hygiene 55, 214-218 (1996).

34. S. D. Melman, M. L. Steinauer, C. Cunningham, L. S. Kubatko, I. N. Mwangi, N. B. Wynn, M. W. Mutuku, D. M. S. Karanja, D. G. Colley, C. L. Black, W. E. Secor, G. M. Mkoji, E. S. Loker, Reduced susceptibility to praziquantel among naturally occurring Kenyan isolates of Schistosoma mansoni., PLoS neglected tropical diseases 3, e504 (2009).

35. S. William, S. Botros, M. Ismail, A. Farghally, T. A. Day, J. L. Bennett, Praziquantel-induced tegumental damage in vitro is diminished in schistosomes derived from praziquantel-resistant infections., Parasitology 122 Pt 1 , 63-66 (2001).

36. N. Kittur, C. H. Campbell, S. Binder, Y. Shen, R. E. Wiegand, J. R. Mwanga, S. M. Kinung'hi, R. M. Musuva, M. R. Odiere, S. H. Matendechero, S. Knopp, D. G. Colley, Discovering, defining, and summarizing persistent hotspots in SCORE studies., The American journal of tropical medicine and hygiene 103, 24-29 (2020).

37. P. A. Mawa, J. Kincaid-Smith, E. M. Tukahebwa, J. P. Webster, S. Wilson, Schistosomiasis morbidity hotspots: Roles of the human host, the parasite and their interface in the development of severe morbidity., Frontiers in immunology 12, 635869 (2021).

38. T. Crellen, M. Walker, P. H. L. Lamberton, N. B. Kabatereine, E. M. Tukahebwa, J. A. Cotton, J. P. Webster, Reduced efficacy of praziquantel against schistosoma mansoni is associated with multiple rounds of mass drug administration., Clinical infectious diseases : an official publication of the Infectious Diseases Society of America 63, 1151-1159 (2016).

39. A. V. Protasio, I. J. Tsai, A. Babbage, S. Nichol, M. Hunt, M. A. Aslett, N. De Silva, G. S. Velarde, T. J. C. Anderson, R. C. Clark, C. Davidson, G. P. Dillon, N. E. Holroyd, P. T. LoVerde, C. Lloyd, J. McQuillan, G. Oliveira, T. D. Otto, S. J. Parker-Manuel, M. A. Quail, R. A. Wilson, A. Zerlotini, D. W. Dunne, M. Berriman, A systematically improved high quality genome and transcriptome of the human blood fluke Schistosoma mansoni., PLoS Negl Trop Dis 6, e1455 (2012).

40. T. J. C. Anderson, P. T. LoVerde, W. Le Clec'h, F. D. Chevalier, Genetic crosses and linkage mapping in schistosome parasites., Trends Parasitol 34, 982-996 (2018).

41. S. K. Volkman, J. Herman, A. K. Lukens, D. L. Hartl, Genome-wide association studies of drug-resistance determinants., Trends in parasitology 33, 214-230 (2017).

42. J. Wit, C. M. Dilks, E. C. Andersen, Complementary approaches with free-living and parasitic nematodes to understanding anthelmintic resistance., Trends in parasitology 37, 240-250 (2021).

43. R. H. Fetterer, R. A. Pax, J. L. Bennett, Praziquantel, potassium and 2,4-dinitrophenol: analysis of their action on the musculature of Schistosoma mansoni., European journal of pharmacology 64, 31-38 (1980).

44. S. Howe, D. Zöphel, H. Subbaraman, C. Unger, J. Held, T. Engleitner, W. H. Hoffmann, A. Kreidenweiss, Lactate as a novel quantitative measure of viability in Schistosoma mansoni drug sensitivity assays., Antimicrobial agents and chemotherapy 59, 1193-1199 (2015). 

available under aCC-BY-NC 4.0 International license.

45. M. Girardot, E. Bayet, J. Maurin, P. Fort, P. Roux, P. Raynaud, SOX9 has distinct regulatory roles in alternative splicing and transcription., Nucleic acids research 46, 9106-9118 (2018).

46. G. Wendt, L. Zhao, R. Chen, C. Liu, A. J. O’Donoghue, C. R. Caffrey, M. L. Reese, J. J. Collins, A single-cell RNA-seq atlas of , javax.xml.bind.JAXBElement@463bbb2c, identifies a key regulator of blood feeding., Science (New York, N.Y.) 369, 1644-1649 (2020).

47. S. William, A. Sabra, F. Ramzy, M. Mousa, Z. Demerdash, J. L. Bennett, T. A. Day, S. Botros, Stability and reproductive fitness of Schistosoma mansoni isolates with decreased sensitivity to praziquantel., International journal for parasitology 31, 1093-1100 (2001).

48. S. Hernando-Amado, F. Sanz-García, P. Blanco, J. L. Martínez, Fitness costs associated with the acquisition of antibiotic resistance., Essays in biochemistry 61, 37-48 (2017).

49. P. Durão, R. Balbontín, I. Gordo, Evolutionary mechanisms shaping the maintenance of antibiotic resistance., Trends in microbiology 26, 677-691 (2018).

50. S. Nair, X. Li, G. A. Arya, M. McDew-White, M. Ferrari, F. Nosten, T. J. C. Anderson, Fitness costs and the rapid spread of , javax.xml.bind.JAXBElement@527e161e, -C580Y substitutions conferring artemisinin resistance., Antimicrobial agents and chemotherapy 62 (2018), doi:10.1128/AAC.00605-18.

51. W. Le Clec'h, F. D. Chevalier, M. McDew-White, F. Allan, B. L. Webster, A. N. Gouvras, S. Kinunghi, L.-A. Tchuem Tchuenté, A. Garba, K. A. Mohammed, S. M. Ame, J. P. Webster, D. Rollinson, A. M. Emery, T. J. C. Anderson, Whole genome amplification and exome sequencing of archived schistosome miracidia., Parasitology 145, 1739-1747 (2018).

52. F. D. Chevalier, W. Le Clec'h, M. McDew-White, V. Menon, M. A. Guzman, S. P. Holloway, X. Cao, A. B. Taylor, S. Kinung'hi, A. N. Gouvras, B. L. Webster, J. P. Webster, A. M. Emery, D. Rollinson, A. Garba Djirmay, K. M. Al Mashikhi, S. Al Yafae, M. A. Idris, H. Moné, G. Mouahid, P. J. Hart, P. T. LoVerde, T. J. C. Anderson, Oxamniquine resistance alleles are widespread in Old World Schistosoma mansoni and predate drug deployment., PLoS pathogens 15, e1007881 (2019).

53. D. Ménard, N. Khim, J. Beghain, A. A. Adegnika, M. Shafiul-Alam, O. Amodu, G. Rahim-Awab, C. Barnadas, A. Berry, Y. Boum, M. D. Bustos, J. Cao, J.-H. Chen, L. Collet, L. Cui, G.-D. Thakur, A. Dieye, D. Djallé, M. A. Dorkenoo, C. E. Eboumbou-Moukoko, F.-E.-C. J. Espino, T. Fandeur, M.-F. Ferreira-da-Cruz, A. A. Fola, H.-P. Fuehrer, A. M. Hassan, S. Herrera, B. Hongvanthong, S. Houzé, M. L. Ibrahim, M. Jahirul-Karim, L. Jiang, S. Kano, W. Ali-Khan, M. Khanthavong, P. G. Kremsner, M. Lacerda, R. Leang, M. Leelawong, M. Li, K. Lin, J.-B. Mazarati, S. Ménard, I. Morlais, H. Muhindo-Mavoko, L. Musset, K. Na-Bangchang, M. Nambozi, K. Niaré, H. Noedl, J.-B. Ouédraogo, D. R. Pillai, B. Pradines, B. Quang-Phuc, M. Ramharter, M. Randrianarivelojosia, J. Sattabongkot, A. Sheikh-Omar, K. D. Silué, S. B. Sirima, C. Sutherland, D. Syafruddin, R. Tahar, L.-H. Tang, O. A. Touré, P. Tshibangu-wa-Tshibangu, I. Vigan-Womas, M. Warsame, L. Wini, S. Zakeri, S. Kim, R. Eam, L. Berne, C. Khean, S. Chy, M. Ken, K. Loch, L. Canier, V. Duru, E. Legrand, J.-C. Barale, B. Stokes, J. Straimer, B. Witkowski, D. A. Fidock, C. Rogier, P. Ringwald, F. Ariey, O. Mercereau-Puijalon, K. A. R. M. A. Consortium, A worldwide map of plasmodium falciparum k13-propeller polymorphisms., The New England journal of medicine 374, 2453-2464 (2016).

54. M. Mayxay, S. Nair, D. Sudimack, M. Imwong, N. Tanomsing, T. Pongvongsa, S. Phompida, R. Phetsouvanh, N. J. White, T. J. C. Anderson, P. N. Newton, Combined molecular and clinical assessment of Plasmodium falciparum antimalarial drug resistance in the Lao People's Democratic Republic (Laos)., The American journal of tropical medicine and hygiene 77, 36-43 (2007).

55. T. O. Apinjoh, A. Ouattara, V. P. K. Titanji, A. Djimde, A. Amambua-Ngwa, Genetic diversity and drug resistance surveillance of Plasmodium falciparum for malaria elimination: is there an ideal tool for resource-limited sub-Saharan Africa?, Malaria journal 18, 217 (2019). 
56. A. C. Kotze, R. K. Prichard, Anthelmintic resistance in haemonchus contortus: History, mechanisms and diagnosis., Advances in parasitology 93, 397-428 (2016).

57. L. A. Melville, E. Redman, A. A. Morrison, P. C. Rebecca Chen, R. Avramenko, S. Mitchell, J. Van Dijk, G. Innocent, F. Sargison, C. Aitken, J. S. Gilleard, D. J. Bartley, Large scale screening for benzimidazole resistance mutations in Nematodirus battus, using both pyrosequence genotyping and deep amplicon sequencing, indicates the early emergence of resistance on UK sheep farms., International journal for parasitology. Drugs and drug resistance 12, 68-76 (2020).

58. C. L. L. Valentim, D. Cioli, F. D. Chevalier, X. Cao, A. B. Taylor, S. P. Holloway, L. Pica-Mattoccia, A. Guidi, A. Basso, I. J. Tsai, M. Berriman, C. Carvalho-Queiroz, M. Almeida, H. Aguilar, D. E. Frantz, P. J. Hart, P. T. LoVerde, T. J. C. Anderson, Genetic and molecular basis of drug resistance and species-specific drug action in schistosome parasites., Science (New York, N.Y.) 342, 1385-1389 (2013).

59. F. D. Chevalier, W. Le Clec'h, N. Eng, A. R. Rugel, R. R. de Assis, G. Oliveira, S. P. Holloway, X. Cao, P. J. Hart, P. T. LoVerde, T. J. C. Anderson, Independent origins of loss-of-function mutations conferring oxamniquine resistance in a Brazilian schistosome population., International journal for parasitology 46, 417-424 (2016).

60. G. Mouahid, M. A. Idris, O. Verneau, A. Théron, M. M. A. Shaban, H. Moné, A new chronotype of lemphSchistosoma mansoni: adaptive significance., Trop Med Int Health 17, 727-732 (2012).

61. R. K. Assaré, R. N. N'Tamon, L. G. Bellai, J. A. Koffi, T.-B. I. Mathieu, M. Ouattara, E. Hürlimann, J. T. Coulibaly, S. Diabaté, E. K. N'Goran, J. Utzinger, Characteristics of persistent hotspots of Schistosoma mansoni in western Côte d'Ivoire., Parasites \& vectors 13, 337 (2020).

62. K. M. Bonner, C. J. Bayne, M. K. Larson, M. S. Blouin, Effects of Cu/Zn superoxide dismutase (SOD1) genotype and genetic background on growth, reproduction and defense in Biomphalaria glabrata., PLoS Negl Trop Dis 6, e1701 (2012).

63. F. A. Lewis, M. A. Stirewalt, C. P. Souza, G. Gazzinelli, Large-scale laboratory maintenance of Schistosoma mansoni, with observations on three schistosome/snail host combinations., J Parasitol 72, 813-829 (1986).

64. D. Cioli, L. Pica-Mattoccia, R. Moroni, Schistosoma mansoni: hycanthone/oxamniquine resistance is controlled by a single autosomal recessive gene., Experimental parasitology 75, 425-432 (1992).

65. R. H. Duvall, W. B. DeWitt, An improved perfusion technique for recovering adult schistosomes from laboratory animals., The American journal of tropical medicine and hygiene 16, 483-486 (1967).

66. W. Le Clec'h, R. Diaz, F. D. Chevalier, M. McDew-White, T. J. C. Anderson, Striking differences in virulence, transmission and sporocyst growth dynamics between two schistosome populations., Parasites \& vectors 12, 485 (2019).

67. H. Li, R. Durbin, Fast and accurate short read alignment with Burrows-Wheeler transform., Bioinformatics (Oxford, England) 25, 1754-1760 (2009).

68. H. Li, B. Handsaker, A. Wysoker, T. Fennell, J. Ruan, N. Homer, G. Marth, G. Abecasis, R. Durbin, 1000 Genome Project Data Processing Subgroup, The sequence Alignment/Map format and SAMtools., Bioinformatics (Oxford, England) 25, 2078-2079 (2009).

69. M. A. DePristo, E. Banks, R. Poplin, K. V. Garimella, J. R. Maguire, C. Hartl, A. A. Philippakis, G. del Angel, M. A. Rivas, M. Hanna, A. McKenna, T. J. Fennell, A. M. Kernytsky, A. Y. Sivachenko, K. Cibulskis, S. B. Gabriel, D. Altshuler, M. J. Daly, A framework for variation discovery and genotyping using next-generation DNA sequencing data., Nature genetics 43, 491-498 (2011). 
70. A. McKenna, M. Hanna, E. Banks, A. Sivachenko, K. Cibulskis, A. Kernytsky, K. Garimella, D. Altshuler, S. Gabriel, M. Daly, M. A. DePristo, The genome analysis toolkit: a MapReduce framework for analyzing nextgeneration DNA sequencing data., Genome research 20, 1297-1303 (2010).

71. J. Köster, S. Rahmann, Snakemake-a scalable bioinformatics workflow engine., Bioinformatics (Oxford, England) 34, 3600 (2018).

72. F. D. Chevalier, W. Le Clec'h, A. C. Alves de Mattos, P. T. LoVerde, T. J. C. Anderson, Real-time PCR for sexing Schistosoma mansoni cercariae., Mol Biochem Parasitol 205, 35-38 (2016).

73. A. M. Emery, F. E. Allan, M. E. Rabone, D. Rollinson, Schistosomiasis collection at NHM (SCAN)., Parasites \& vectors 5, 185 (2012).

74. R Core Team, R: A language and environment for statistical computing, R Foundation for Statistical Computing - Vienna, Austria (2018).

75. M. O.J., PerlPrimer: cross-platform, graphical primer design for standard, bisulphite and real-time PCR., Bioinformatics 20, 2471-2 (2004).

76. G. Krautz-Peterson, R. Bhardwaj, Z. Faghiri, C. A. Tararam, P. J. Skelly, RNA interference in schistosomes: machinery and methodology., Parasitology 137, 485-495 (2010).

77. R. Bhardwaj, G. Krautz-Peterson, P. J. Skelly, Using RNA interference in schistosoma mansoni., Methods in molecular biology (Clifton, N.J.) 764, 223-239 (2011).

78. X. Rao, X. Huang, Z. Zhou, X. Lin, An improvement of the $2^{\wedge}(-$ delta delta CT) method for quantitative real-time polymerase chain reaction data analysis., Biostatistics, bioinformatics and biomathematics 3, 71-85 (2013).

79. G. Krautz-Peterson, R. Bhardwaj, Z. Faghiri, C. A. Tararam, P. J. Skelly, RNA interference in schistosomes: machinery and methodology., Parasitology 137, 485-495 (2010).

80. W. Le Clec'h, R. Diaz, F. D. Chevalier, M. McDew-White, T. J. C. Anderson, Striking differences in virulence, transmission and sporocyst growth dynamics between two schistosome populations., Parasites \& vectors 12, 485 (2019).

Acknowledgments: We thank the Vivarium of the Southwest National Primate Research Center (SNPRC) for providing rodent care. This research was supported by a Cowles fellowship (WLC) from Texas Biomedical Research Institute (13-1328.021), and NIH R01AI133749 (TJCA) and was conducted in facilities constructed with support from Research Facilities Improvement Program grant C06 RR013556 from the National Center for Research Resources. SNPRC research at Texas Biomedical Research Institute is supported by grant P51 OD011133 from the 570 Office of Research Infrastructure Programs, NIH. Miracidia samples for sequence analysis were provided by Schistosomiasis Collection at the Natural History Museum (SCAN) funded by the Wellcome Trust (grant 104958/Z/14/Z) and curated Muriel 
Rabone. We thank Dr Oumar T. Diaw and Moumoudane M. Seye (Institut Sénégalais de Recherches Agricoles, Senegal) for the collections in Senegal (EU-CONTRAST project); Dr Anouk Gouvras (Global Schistosomiasis Alliance (GSA)), Mariama Lamine and the Re'seau International Schistosomiases Environnemental Aménagement et Lutte (RISEAL) team (Niger) for the collections in Niger; Dr Anouk Gouvras (GSA) and the National Institute for Medical Research (NIMR, Mwanza Tanzania) team Dr Teckla Angelo, Honest Nagai, Boniface Emmanuel, John Igogote, Sarah Buddenborg and Reuben Jonathan for the collections in Tanzania as part of the Schistosomiasis Consortium for Operational Research and Evaluation (SCORE) project (https://score.uga.edu).

\section{Funding:}

National Institutes of Health grant 1R01AI123434 (TJCA, PTL)

National Institutes of Health grant 1R01AI133749 (TJCA)

National Institutes of Health grant NIH R01AI145871 (JSM)

Ministry of Health in Oman, the Sultan Qaboos University (grant no. IG/ MED/MICR/00/01)

French Ministry of Foreign Affairs (French Embassy in Oman) (grants nos. 402419B, 402415K and 339660F)

CNRS- Sciences de la Vie (grants no. 01N92/0745/1 and 02N60/1340)

CNRS-Direction des Relations internationales (grants no. 01N92/0745 and 02N60/1340)

PICS-CNRS no. 06249: FRANC-INCENSE

\section{Author contributions:}

Conceptualization: WLC, PTL, TJCA

Methodology: WLC, FDC, PTL, JM

Investigation: WLC, FDC, ACM, AS, RD, JM

Visualization: WLC, FDC

Field samples: HM, GM, MI, KAM, SAY

Funding acquisition: TJCA, PTL, JM, HM, GM 
Project administration: TJCA, PTL

Supervision: TJCA, WLC, FDC, PTL, JM

Writing - original draft: TJCA, WLC, FDC

Competing interests: Authors declare that they have no competing interests.

\section{Data and materials availability:}

Sequence data from field collected S. mansoni is available from: PRJNA439266, PRJNA560070,

Code is available from: https://github.com/fdchevalier/PZQ-R_DNA, https://github.com/fdchevalier/PZQ- 


\section{File S1.}

\section{EXPANDED MATERIALS AND METHODS}

\section{Study design}

This study was designed to determine the genetic basis of PZQ-R, and was stimulated by the initial observation that a laboratory $S$. mansoni population generated through selection with PZQ contained both PZQ-S and PZQ-R individuals. The project had 6 stages:(i) QTL location. We conducted a genome-wide association study (GWAS). This involved measuring the PZQ-response of individual worms, pooling those showing high levels of resistance and low levels of resistance, sequencing the pools to high read depth, and then identifying the genome regions showing significant differences in allele frequencies between high and low resistance parasites. (ii) Fine mapping of candidate genes. We identified potential candidate genes in these QTL regions, through examination of gene annotations, and exclusion of genes that are not expressed in adults. We also determined whether the loci determining PZQ-R are inherited in a recessive, dominant or co-dominant manner (iii) Marker assisted purification of PZQ-S and PZQ-R parasites. To separate PZQR and PZQ-S parasites into "pure" populations, we genotyped larval parasites for genetic markers in the QTL regions and infected rodents with genotypes associated with PZQ-R or PZQ-S. To verify that this approach worked, we then measured the $\mathrm{IC}_{50}$ for each of the purified populations. (iv) Characterization of purified SmLE-PZQ-ER and SmLE-PZQ-ES populations. Separation of SmLE-PZQ-ES and SmLE-PZQ-

ER parasite populations allowed us to characterize these in more detail. Specifically, we measured expression in juvenile and adult worms of both sexes in both SmLE-PZQ-ES and SmLE-PZQ-ER parasites. We also quantified parasite fitness traits. (v) Functional analysis. We used RNAi and chemical manipulation approaches to modulate activity of candidate genes and determine the impact of PZQ-resistance. We also used transient expression of candidate genes in cultured mammalian cells, to determine the impact of particular SNPs on response to PZQ-exposure. (vi) Survey of PZQ-resistance variants in field collected parasites. Having determined the gene underlying PZQ-R in laboratory parasites, we examined sequence 
variation in this gene in a field collection of $S$. mansoni parasites collected to examine the frequency of sequence variants predicted to result in PZQ-resistance.

\section{Ethics statement}

This study was performed in accordance with the Guide for the Care and Use of Laboratory Animals of the National Institutes of Health. The protocol was approved by the Institutional Animal Care and Use Committee of Texas Biomedical Research Institute (permit number: 1419-MA and 1420-MU). Details of ethical permission for collection of samples from humans are described in Chevalier et al. (51, 52).

\section{Biomphalaria glabrata snails and Schistosoma mansoni parasites}

Uninfected inbred albino Biomphalaria glabrata snails (line Bg121 derived from 13-16-R1 line (62)) were reared in 10-gallon aquaria containing aerated freshwater at $26-28^{\circ} \mathrm{C}$ on a $12 \mathrm{~L}-12 \mathrm{D}$ photocycle and fed $\mathrm{ad}$ libitum on green leaf lettuce. All snails used in this study had a shell diameter between 8 and $10 \mathrm{~mm}$. We used inbred snails to minimize the impact of snail host genetic background on the parasite life history traits (66).

The SmLE schistosome (Schistosoma mansoni) population was originally obtained from an infected patient in Belo Horizonte (Minas Gerais, Brazil) in 1965 and has since been maintained in laboratory (63), using B. glabrata NMRI, inbred Bg36 and Bg121 population as intermediate snail host and Syrian golden hamsters (Mesocricetus auratus) as definitive hosts. The SmLE-PZQ-R schistosome population was generated in Brazil by applying a single round of PZQ selection pressure on SmLE parasites at both snail and rodent stages (26). The SmLE-PZQ-R population has been maintained in our laboratory using B. glabrata NMRI, Bg36 and Bg121 snail population and hamsters as the definitive host since 2014 .

\section{Drug resistance tests:}

Dose-response curves to PZQ in SmLE and SmLE-PZQ-R populations 
Drug sensitivity to Praziquantel (PZQ) was initially measured using a modified protocol (64) in SmLE and SmLE-PZQ-R parasite populations. Ten adult males, recovered by perfusion from infected hamsters $(\mathrm{t}+45$ days post-infection) (65) from SmLE or SmLE-PZQ-R population were placed into each well of a 24-well microplate containing $1 \mathrm{~mL}$ of High glucose DMEM supplemented with $15 \%$ heat-inactivated fetal bovine serum, $100 \mathrm{U} / \mathrm{mL}$ penicillin and $100 \mu \mathrm{g} / \mathrm{mL}$ streptomycin (DMEM complete media). We performed control and experimental groups in triplicate ( $N=240 \mathrm{worms} /$ parasite populations). We exposed adult worms to PZQ $(0.1 \mu \mathrm{g} / \mathrm{mL}, 0.3 \mu \mathrm{g} / \mathrm{mL}, 0.9 \mu \mathrm{g} / \mathrm{mL}, 2.7 \mu \mathrm{g} / \mathrm{mL}, 8.1 \mu \mathrm{g} / \mathrm{mL}, 24.3 \mu \mathrm{g} / \mathrm{mL}$ and $72.9 \mu \mathrm{g} / \mathrm{mL})$ for $24 \mathrm{~h}$. Worms were then washed three times in drug-free medium and incubated $\left(37^{\circ} \mathrm{C}, 5 \% \mathrm{CO}_{2}\right)$ for 2 days. Control groups were exposed only to the drug diluent dimethyl sulfoxide (DMSO). The parasites were observed daily under a stereomicroscope for the 5 days of the experiment and the number of dead worms visually scored. Worms were defined as "dead" if they showed no movements and became opaque. We scored PZQ-resistance as a binary trait: parasites that recovered were classed as resistant, while parasites that failed to recover were classed as sensitive.

\section{Metabolic assessment of worm viability using L-lactate assay}

We adapted a method for metabolic assessment of worm viability using L-lactate assay (44). Briefly, adult male SmLE-PZQ-R worms recovered from infected hamsters were placed individually in 96-well plates containing $100 \mu \mathrm{m}$ mesh filter insert (Millipore) in $250 \mu \mathrm{L}$ DMEM complete media, and allowed to adapt for $24 \mathrm{~h}$. We added PZQ (24.3 $\mu \mathrm{g} / \mathrm{mL}$ in DMEM complete media) for the PZQ treated group, while controls were treated with the same volume of drug diluent DMSO. We also added a heat-killed worm control group: adult male worms were placed into a microfuge tube containing distilled water and heated in a dry bath $\left(80^{\circ} \mathrm{C}, 15 \mathrm{~min}\right)$, and then plated in $96-$ well plate with $100 \mu \mathrm{m}$ mesh insert. Drug resistance test was conducted as described above (see Dose-response curves to PZQ in SmLE and SmLE-PZQ-R populations). At $48 \mathrm{~h}$ post-treatment, the supernatant $(125 \mu \mathrm{L})$ was collected from each well and immediately stored at $80{ }^{\circ} \mathrm{C}$ until processing. 
We measured lactate levels in the supernatants of in vitro treated adult male worms with a

colorimetric L-lactate assay kit (Sigma) using 96-well, optical clear-bottom plates (Corning) following the manufacturer's specifications, with minor modifications. Briefly, $5 \mu \mathrm{L}$ of supernatant were diluted into 20 $\mu \mathrm{L}$ of $\mathrm{ddH}_{2} \mathrm{O}$ to fit within the linear range of the assay. We then combine $24 \mu \mathrm{L}$ of the assay buffer to $1 \mu \mathrm{L}$ of diluted supernatant (1/5 dilution) in each test well. and added $25 \mu \mathrm{L}$ of the lactate reaction mix (24 $\mu \mathrm{L}$ of the assay buffer, $0.5 \mu \mathrm{L}$ of enzyme mix and $0.5 \mu \mathrm{L}$ of lactate assay probe $-\mathrm{V}_{\text {total }}=50 \mu \mathrm{L} /$ well). We also made a lactate standard curve to allow accurate lactate quantification in worm supernatants. After 45 min of incubation in the dark at room temperature, the plate was read by a spectrophotometer (Molecular Devices) at $570 \mathrm{~nm}$. Lactate quantities in worm supernatant were assessed following the manufacturer's instruction, taking in account our dilution factor. All measurement series included a DMEM complete media control to determine the background lactate level, which was then subtracted from the lactate quantity of the respective measurements.

\section{Genome wide association analysis and QTL mapping}

\section{$\underline{\text { Schistosome infections }}$}

Eggs were collected from livers of hamster infected with SmLE-PZQ-R and hatched under light for $30 \mathrm{~min}$ in freshwater to obtain miracidia (66). We then exposed one thousand Bg121 snails to five miracidia/snail. After 30 days, snails were individually exposed to light in 24-well plates to shed cercariae. Eight hamsters were exposed to 840 cercariae ( 4 cercariae/snail) from a batch of 210 shedding snails. We euthanized hamsters after 45 days to collect adult worms.

\section{Phenotypic selection}

Adult SmLE-PZQ-R worms were collected, separated by sex and males were plated individually in 96-well plates (60 worms per plate) containing 100uM mesh filter insert (Millipore) in $250 \mu \mathrm{L}$ of DMEM complete media and treated with a dose of $24.3 \mu \mathrm{g} / \mathrm{mL}$ PZQ as describe above (i.e. Metabolic assessment of worms viability using L-lactate assay). A group of 12 worms were treated with the same volume of drug diluent 
DMSO. This GWAS experiment was done twice independently. A total of 590 and 691 adult male worms were collected, cultured in vitro and exposed to PZQ for the two experiments respectively.

Worm media supernatants $(125 \mu \mathrm{L})$ were collected in 96-well PCR plates after $24 \mathrm{~h}$ in culture (to assess the viability of the worms before PZQ treatment - adult male worms should release $\geq 40 \mathrm{nmol} / \mathrm{h}$ of lactate in supernatant) and $48 \mathrm{~h}$ post-treatment (to assess their viability after PZQ treatment). Plates containing supernatant were immediately stored at $-80{ }^{\circ} \mathrm{C}$ until processing. Lactate levels in supernatants were quantified as described above (see Metabolic assessment of worm viability using L-lactate assay). We phenotype the worms and categorize them into two groups: i) Recovered worms (i.e. releasing $\geq 40 \mathrm{nmol} / \mathrm{h}$ of lactate in supernatant) and ii) Susceptible worms (i.e. releasing less $40 \mathrm{nmol} / \mathrm{h}$ of lactate in supernatant). Among these two groups, we took the $20 \%$ of the treated worms releasing in their media the highest amount of lactate (average L-lactate production $\pm \mathrm{SD}$ : Experiment $1=61.44 \mathrm{nmol} / \mathrm{h} \pm 13.16 /$ Experiment $2=56.38$ $\mathrm{nmol} / \mathrm{h} \pm 10.82$ ) and the $20 \%$ of the treated worms releasing the lowest amount of lactate (average L-lactate production $\pm \mathrm{SD}$ : Experiment $1=28.61 \mathrm{nmol} / \mathrm{h} \pm 5.32 /$ Experiment $2=23.04 \mathrm{nmol} / \mathrm{h} \pm 4.14), 48 \mathrm{~h}$ post PZQ treatment respectively.

\section{$\underline{D N A}$ extraction and library preparation}

We sequenced whole genomes of the two pools of recovered (i.e. resistant to PZQ, Experiment 1: 116 worms / Experiment 2: 137 worms) and susceptible worms (Experiment 1: 116 worms / Experiment 2: 137 worms). We then estimated allele frequencies in each pool to identify genome regions showing high differentiation.

a. gDNA extraction: We extracted gDNA from pools of worms using the Blood and Tissue kit (Qiagen). We homogenized worms in DNA extraction kit lysis buffer using sterile micro pestles., incubated homogenates $\left(56^{\circ} \mathrm{C}, 2\right.$ hour) and recovered gDNA in $200 \mu \mathrm{L}$ of elution buffer. We quantified the worm gDNA recovered using the Qubit dsDNA HS Assay Kit (Invitrogen).

b. Whole genome library preparation and sequencing: We prepared whole genome libraries from pools of worm gDNA in triplicate using the KAPA HyperPlus kit (KAPA Biosystems) according to 
the manufacturer's protocol. For each library, we sheared $100 \mathrm{ng}$ of gDNA by adaptive focused acoustics (Duty factor: 10\%; Peak Incident Power: 175; Cycles per Burst: 200; Duration: 180 seconds) in AFA tubes (Covaris S220 with SonoLab software version 7 (Covaris, Inc., USA)) to recover fragmented DNA (150-200 bp). Library indexing was done using KAPA Dual Adapters at $15 \mu \mathrm{M}$ for $1 \mathrm{~h}$. We used $6 \mathrm{PCR}$ cycles for post-ligation library amplification. We performed size selection on the indexed-amplified libraries using KAPA Pure bead (0.7x first upper size cut; 0.9x second lower size cut). We quantified libraries by qPCR using KAPA library quantification kit (KAPA Biosystems) and their respective fragment size distribution was assessed by TapeStation (Agilent). We sequenced the libraries on a HiSeq X sequencer (Illumina) using 150 bp pair-end reads. Raw sequence data are available at the NCBI Sequence Read Archive (PRJNA699326).

\section{Bioinformatic analysis}

Jupyter notebook and scripts used for processing the sequencing data and identifying the QTL are available on Github (https://github.com/fdchevalier/PZQ-R_DNA).

a. Sequence analysis and variant calling: We aligned the sequencing data against the S. mansoni reference genome (schistosoma_mansoni.PRJEA36577.WBPS14) using BWA (v0.7.17) (67) and SAMtools (v1.10) (68). We used GATK (v4.1.8.1) $(69,70)$ to mark PCR duplicates and recalibrate base scores. We used the HaplotypeCaller module of GATK to call variants (SNP/indel) and the GenotypeGVCFs module to perform a joint genotyping on each chromosome or unassembled scaffolds. We merged VCF files using the MergeVcfs module. All these steps were automatized using Snakemake (v5.14.0) (71).

b. QTL identification: We expect the genome region underlying resistance to be enriched in variants from high lactate producing worms. To evaluate statistical evidence for such enrichment, we examined the difference in allele frequencies between low and high lactate parasites across the genome by calculating a $Z$-score at each bi-allelic site. To minimize bias, we weighed $Z$-scores by including the number of worms in each treatment and the difference in the total read depth across 
the triplicated libraries of each treatment at the given variant. We calculated $Z$-scores for each biological replicate as follows:

$$
Z=\frac{p_{1}-p_{2}}{\sqrt{p_{0}\left(1-p_{0}\right)\left(\frac{1}{x \cdot n_{1}}+\frac{1}{x \cdot n_{2}}+\frac{1}{d_{1}}+\frac{1}{d_{2}}\right)}}
$$

where $p 1$ and $p 2$ are the estimated allele frequencies in the low and high lactate parasites pools, respectively; $p 0$ is the allele frequency under the null hypothesis $\mathrm{H} 0: p 1=p 2$ estimated from the average of $p 1$ and $p 2 ; n 1$ and $n 2$ are the number of worms in the low and high lactate parasites pools, respectively, factor $x$ for each $n$ reflecting the ploidy state $(x=2)$; and $d 1$ and $d 2$ are the sequencing depths for the low and high lactate parasite pools, respectively.

We combined $Z$-scores generated from each biological replicate as follows:

$$
Z_{c}=\frac{Z_{1}+Z_{2}}{\sqrt{2}}
$$

where $Z_{1}$ and $Z_{2}$ were $Z$-scores from replicate 1 and 2 , respectively. The p-values were obtained by comparing the negative absolute value of $Z$-scores to the standard normal distribution. To determine the significant threshold, Bonferroni correction was calculated with $\alpha=0.05$. These analyses are available in the Jupyter notebook and associated scripts (https://github.com/fdchevalier/PZQ-R_DNA).

\section{Relationship between worm genotype at Chr 2 and 3 and PZQ-R phenotype}

To validate the impact of worm genotypes on its PZQ resistance phenotypes and determine whether PZQR shows recessive, dominant or codominant inheritance, we determined the PZQ-R phenotype of individual worms, which were then genotyped for markers at the peak of the QTLs located.

\section{$\underline{\text { Measuring } P Z Q-R \text { in individual worms }}$}


We collected 120 SmLE-PZQ-R adult male worms, plated them individually in 96-well plates containing a mesh filter insert, cultured them in vitro, treated them with PZQ $(24.3 \mu \mathrm{g} / \mathrm{mL})$ and collected media supernatants before (after $24 \mathrm{~h}$ in culture) and $48 \mathrm{~h}$ post-treatment, and used L-lactate assays to determine PZQ-R status (see Phenotypic selection). We extracted gDNA from each worm individually. Briefly, we transferred worms into 96-well PCR plates, added $100 \mu \mathrm{L}$ of $6 \%$ Chelex ${ }^{\circledR}$ solution containing $1 \%$ Proteinase $\mathrm{K}(20 \mathrm{mg} / \mathrm{mL})$, incubated for $2 \mathrm{~h}$ at $56{ }^{\circ} \mathrm{C}$ and $8 \mathrm{~min}$ at $100{ }^{\circ} \mathrm{C}$, and transferred the supernatant containing worm gDNA into fresh labeled 96-well plates.

\section{PCR-RFLP conditions for Chr. 2 and Chr. 3 loci}

We used PCR-RFLP to genotype single worms at loci marking QTL peaks on chr. 2 (C>A, chr SMV7_2: 1072148) and chr. 3 (T>C, chr SMV7_3: 741987). Primers were designed using PerlPrimer v1.21.1 (75) (Table S4). We digested PCR amplicons for chr. 2 with BslI (NEB) and chr. 3 with Mse1 (NEB), and visualized digested PCR amplicons by $2 \%$ agarose gel electrophoresis.

\section{Quantitative PCR validation of copy number variation (CNV) in single worms}

We genotyped each individual worm for a deletion identified on chr. 3 at position $1220683-1220861 \mathrm{bp}$ using a custom quantitative PCR assay. This was done to examine the association between deletion of this genomic region and PZQ resistant genotype. We quantified the copy number in this region relative to a single copy gene from S. mansoni ( $\alpha$-tubulin 2, LeClech2019). The CNV genotype for each parasite corresponds to the ratio of the $\mathrm{CNV}$ copy number and the $\alpha$-tubulin 2 gene copy number: $0=$ complete deletion, $0.5=$ one copy, $1=$ two copies. Methods and primers are described in Table S4. We then compared individual worm phenotypes for each of the three $\mathrm{CNV}$ genotypes $(0,1$ or 2 copies) to determine the association between CNV and PZQ response.

\section{Marker assisted selection of resistant and susceptible parasite populations}

\section{Selection of SmLE-PZQ-ER and SmLE-PZQ-ES populations}


We separated the polymorphic SmLE-PZQ-R schistosome population based on chr. 3 QTL genotype using the PCR-RFLP as described. We exposed 960 inbred B. glabrata Bg121 snails to one miracidium SmLEPZQ-R (66). At four weeks post-exposure, we identified infected snail $(N=272)$, and collected cercariae from individual snails. We extracted cercarial DNA using 6\%Chelex (66)), and genotyped each parasite for chr. 3 locus using our PCR-RFLP (Homozygous R/R: $n=89-36 \%$; Homozygous S/S: $n=39-16 \%$; Heterozygous R/S: $n=117-49 \%$ ) and determine their gender by PCR (72). We selected $32 \mathrm{R} / \mathrm{R}$ parasites (homozygous for the Sm.TRPM $P Z Q$ resistant-associated allele) and $32 \mathrm{~S} / \mathrm{S}$ genotypes (i.e. homozygous for the $S m . T R P M_{P Z Q}$ sensitive-associated allele). For both R/R and S/S we used 13 males and 19 females. We exposed 5 hamsters to 800 cercariae of $32 \mathrm{R} / \mathrm{R}$ genotypes parasites and 5 hamsters to 800 cercariae $32 \mathrm{~S} / \mathrm{S}$ genotyped parasites. This single generation marker assisted selection procedure generates two subpopulations: SmLE-PZQ-ER is expected to be enriched for parasites with $\mathrm{R} / \mathrm{R}$ genotype and to show strong PZQ-R, while SmLE-PZQ-ES is enriched for S/S genotypes and is expected to be highly sensitive to PZQ).

\section{PZQ IC 50 with SmLE-PZQ-ER and SmLE-PZQ-ES}

Forty-five days after exposure to cercariae, we euthanized and perfused hamsters to recover adult schistosome worms for each of the two subpopulations (SmLE-PZQ-ER and SmLE-PZQ-ES). We separated worms by sex and we set adult males in 96-well plates containing $100 \mu \mathrm{m}$ mesh filter insert (Millipore) and cultured in $250 \mu \mathrm{L}$ DMEM complete media as described.

We determined PZQ dose-response for both SmLE-PZQ-ER and SmLE-PZQ-ES population. We exposed individual worms ( $N=60 /$ population/treatment) to PZQ $(0.1 \mu \mathrm{g} / \mathrm{mL}, 0.3 \mu \mathrm{g} / \mathrm{mL}, 0.9 \mu \mathrm{g} / \mathrm{mL}, 2.7$ $\mu \mathrm{g} / \mathrm{mL}, 8.1 \mu \mathrm{g} / \mathrm{mL}, 24.3 \mu \mathrm{g} / \mathrm{mL}$ and $72.9 \mu \mathrm{g} / \mathrm{mL}$ ) or drug diluent (DMSO control). Worm media supernatants $(125 \mu \mathrm{L})$ were collected in 96-well PCR plates before treatment (after $24 \mathrm{~h}$ in culture) and $48 \mathrm{~h}$ post-treatment. We quantified lactate levels in supernatants described and we assess variation in lactate production for each individual worm. 
gDNA extraction and library preparation

SmLE-PZQ-ER and SmLE-PZQ-ES parasite populations were maintained in our laboratory. We recovered the F1 worms from each populations and extract gDNA from pools of adult males and females separately as described above. We prepared whole genome libraries from these pools in triplicate using the KAPA HyperPlus kit (KAPA Biosystems) as described (see Whole genome library preparation and sequencing). We sequenced the libraries on a HiSeq X sequencer (Illumina) using 150 bp paired-end reads. Sequence data are available at the NCBI Sequence Read Archive (accession numbers PRJNA701978).

\section{$\underline{\text { Bioinformatic analysis }}$}

The analysis was identical to the GWAS and QTL mapping analysis. This can be replicated with the Jupyter notebook and associated scripts (https://github.com/fdchevalier/PZQ-R_DNA).

\section{Transcriptomic analysis of resistant and susceptible schistosome worms to PZQ at juvenile (28 days)}

\section{and adult ( 45 days) stages}

\section{$\underline{\text { Sample collection }}$}

Juvenile and adult $S$. mansoni SmLE-PZQ-ER and SmLE-PZQ-ES worms were recovered by perfusion from hamsters at 28 days (juveniles) or 45 days (adults) post-infection. Worms from each population were placed in DMEM complete media, separated by sex, and aliquoted in sterile RNAse free microtubes which were immediately snap-frozen in liquid nitrogen and stored at $-80{ }^{\circ} \mathrm{C}$ until RNA extractions. For each subpopulation, (SmLE-PZQ-ER or SmLE-PZQ-ES), we collected 3 biological replicates of 30 males and 3 replicates of 30 females for the $28 \mathrm{~d}$ juvenile worms and 3 biological replicates of 30 males and 3 replicates of 60 females for the $45 \mathrm{~d}$ adult worms.

\section{$\underline{R}$ A extraction and RNAseq library preparation}

\section{a. RNA extraction}


1080

We extracted total RNA from all the S. mansoni adult and juvenile worms collected using the RNeasy Mini kit (Qiagen). Samples were randomized prior to RNA extraction to minimize batch effects. We homogenized worms in $600 \mu \mathrm{L}$ RNA lysis buffer (RTL buffer, Qiagen) using sterile micro pestles, followed by passing the worm lysate 10 times through a sterile needle (23 gauge). We recovered total RNA in $25 \mu \mathrm{L}$ elution buffer. We quantified the RNA recovered using the Qubit RNA Assay Kit (Invitrogen) and assessed the RNA integrity by TapeStation (Agilent - RNA integrity numbers of $\sim 8.5-10$ for all the samples).

\section{b. RNAseq library preparation}

We prepared RNAseq libraries using the KAPA Stranded mRNA-seq kit (KAPA Biosystems) using 500ng RNA diluted in 50uL Tris- $\mathrm{HCl}(\mathrm{pH} 8.0)$ for each library. We fragmented mRNA (6 min $\left.94^{\circ} \mathrm{C}\right)$, indexed libraries using $3^{\prime}$-dTMP adapters $\left(7 \mu \mathrm{M}, 1\right.$ hour at $\left.20^{\circ} \mathrm{C}\right)$, and used 6 PCR cycles for post-ligation library amplification. We quantified indexed libraries by qPCR (KAPA library quantification kit (KAPA Biosystems)) and assessed their fragment size distribution by TapeStation (Agilent). We sequenced the libraries on a HiSeq 4000 sequencer (Illumina) using 150 bp pair-end reads, pooling 12 RNAseq libraries/lane. Raw sequence data are available at the NCBI Sequence Read Archive under accession numbers PRJNA704646.

\section{c. Bioinformatic analysis.}

To identify differentially expressed genes between the different groups, we aligned the sequencing data against the S. mansoni reference genome (schistosoma_mansoni.PRJEA36577.WBPS14) using STAR (v2.7.3a) (ref). We quantified gene and isoform abundances by computing transcripts per million values using RSEM (v1.3.3) (ref). We compared these abundances between groups (ES/ER, males/females, juveniles/adults) using the R package EBSeq (v1.24.0) (ref). Jupyter notebooks and associated scripts are available on Github (https://github.com/fdchevalier/PZQ-R_RNA).

\section{Manipulation of $\operatorname{Sm} . T R P M_{P Z O}$ channel expression or function:}

\section{$\underline{R N A \text { interference }}$}


We used RNA interference to knock down the expression of Smp_246790 gene in order to functionally validate the implication of Sm.TRPM $P Z Q$ on schistosome PZQ resistance. SmLE-PZQ-R adult male worms were freshly recovered from infected hamsters and placed in 24-well plates for in vitro culture (10 adult male worms/well).

\section{a. $\quad$ SiRNA treatment on S. mansoni adult male worms}

Small inhibitory RNAs (siRNAs) targeting specific schistosome genes were designed using the on-line IDT RNAi Design Tool (https://www.idtdna.com/Scitools/Applications/RNAi/RNAi.aspx) (Table S2) and synthesized commercially by Integrated DNA Technologies (IDT, Coralville, IA). To deliver the siRNAs, we electroporated schistosome parasites (10 adults/group - each group done in triplicate) in $100 \mu \mathrm{L}$ RPMI medium containing $2.5 \mu \mathrm{g}$ siRNA or the equivalent volume of $\mathrm{ddH}_{2} \mathrm{O}$ (no siRNA control), in a $4 \mathrm{~mm}$ cuvette by applying a square wave with a single $20 \mathrm{~ms}$ impulse, at $125 \mathrm{~V}$ and at room temperature (Gene Pulser Xcell Total System (BioRad)) (76, 77). Parasites were then transferred to $1 \mathrm{~mL}$ of complete DMEM media in 24-well plates. After overnight culture, medium was replaced with fresh DMEM complete media. We measured gene expression by quantitative real-time PCR (RT-qPCR) 2 days after siRNA treatment.

\section{b. $\quad d s R N A$ treatment on $\mathrm{S}$. mansoni adult male worms}

We synthetized double-stranded RNA according to Wang et al., 2020 (Table S2). For dsRNA treatment, 10 adult male worms/group (each group done in triplicate) were cultured in $1 \mathrm{~mL}$ DMEM complete media and treated with 90 ug dsRNA at day 0 , day 1 and day 2 . Media was changed every $24 \mathrm{~h}$ and fresh dsRNA was added. On day 3, we harvested worms and measured gene expression by quantitative real-time PCR (RTqPCR).

\section{c. RNA extraction and gene expression analysis by RT-qPCR}

We extracted total RNA from parasites ( $N=10$ worms/sample) using the RNeasy Mini kit (Qiagen) (see RNA extraction). Complementary DNA (cDNA) was generated from extracted RNA (500 ng - $1 \mu \mathrm{g}$ ) using SuperScript-III and Oligo-dT primers (ThermoFisher). Relative quantification of genes of interest was performed in duplicate by qPCR analysis using QuantStudio 5 System (Applied Biosystems) and SYBR Green master mix (ThermoFisher), compared with a serially diluted standard of PCR products (generated 
from cDNA) for each of the gene tested (66). Standard curves allow evaluating the efficiency of each pairs of primers, for both housekeeping and target genes using QuantStudio Design and Analysis Software. Expression was normalized to SmGAPDH housekeeping gene (Table S2) using the efficiency $\mathrm{E}^{\Delta \Delta \mathrm{Ct}}$ method (78).

\section{Specific Sm.TRPM $\underline{P Z Q}$ chemical inhibitor and activators}

We used specific chemical inhibitor and activators (Chulkov et al., in prep) to manipulate the function of Sm.TRPMPZQ to examine the impact on PZQ-response. We placed individual SMLE-PZQ-ER and SMLEPZQ-ES adult male worms in 96-well plates with $100 \mu \mathrm{m}$ mesh filter insert containing DMEM complete media and cultured described above (see Metabolic assessment of worm viability using L-lactate assay). After $24 \mathrm{~h}$ in culture, 20 worms from each population were treated either with a cocktail combining PZQ (1 $\mu \mathrm{g} / \mathrm{mL}$ ) and i) $50 \mu \mathrm{M}$ of $S m . T R P M_{P Z Q}$ blocker (MB2) or ii) $10 \mu \mathrm{M}, 25 \mu \mathrm{M}$ or $50 \mu \mathrm{M}$ of $\operatorname{Sm} . T R P M_{P Z Q}$ activator (MV1) respectively or iii) drug diluent (DMSO). We also set up control plates to evaluate the impact of $S m . T R P M_{P Z Q}$ blocker or activator alone. In that case, 20 worms from each population were treated with a cocktail combining drug diluent DMSO and Sm.TRPMPZQ blocker (MB2) or activator (MV1) at the same concentrations mentioned above. Worms were exposed to these drug cocktails for $24 \mathrm{~h}$, washed 3 times with drug-free medium, and incubated $\left(37^{\circ} \mathrm{C}, 5 \% \mathrm{CO}_{2}\right)$ for 2 days.

We collected Worm media supernatants $(125 \mu \mathrm{L})$ in $96-$ well PCR plates before treatment (after $24 \mathrm{~h}$ in culture) and $48 \mathrm{~h}$ post-treatment and Lactate levels in supernatants were quantified as described above (see Metabolic assessment of worm viability using L-lactate assay). We used these results to determine the impact of blockers or activators on variation in lactate production.

\section{$\underline{\text { In vivo parasite survival after } P Z Q \text { treatment }}$}

We used 24 female Balb/C mice (purchased from Envigo at 6 weeks-old and housed in our facility for one week before use) split into two groups: one group exposed by tail immersion to SmLE-PZQ-ER (80 cercariae/mouse from 40 infected snails) and the second one to SmLE-PZQ-ES (80 cercariae/mouse from 
40 infected snails). Each mouse was identified by a unique tattoo ID and an ear punch for assessing treatment status (PZQ or drug diluent control). Immediately after infection, we stained the content of each infection vial with $10 \mu \mathrm{L} 0.4 \%$ Trypan blue and counted all the cercarial tails/heads or complete cercariae to determine the cercarial penetration rate for each mouse. We kept infected mice in 4 cages (2 cages/parasite populations and 6 animals per cage) at $21-22^{\circ} \mathrm{C}$ and $39 \%-50 \%$ humidity and monitored them daily.

Forty days post-infection, we weighed mice and treated them by oral gavage with either $120 \mathrm{mg} / \mathrm{kg}$ of PZQ (diluted in 1\% DMSO + vegetable oil - Total volume given/mouse: $150 \mu \mathrm{L}$ ) or the same volume of drug diluent only (control group). To minimize batch effects, 3 mice were treated with PZQ and 3 with the drug diluent per cage for each parasite group (SmLE-PZQ-ER or SmLE-PZQ-ES). Mice were monitored daily until euthanasia and perfusion (65), at day 50 post-infection. We recorded the weight of each mouse before euthanasia. After euthanasia and perfusion, we also weighted the liver and spleen of each individual. We carefully recovered worms from the portal vein, liver and intestine mesenteric venules of each mouse. Worms were separated by sex and counted.

\section{$\underline{\text { Sm.TRPM }}{ }_{P Z O}$ variants in $S$. mansoni field samples}

\section{Variants identification in exome-sequenced data from natural S. mansoni parasites}

We utilized exome sequence data from S. mansoni from Africa, South America and the middle East to investigate variation in Sm.TRPMPZQ. African miracidia were from the Schistosomiasis collection at the Natural History Museum (SCAN) (73), Brazilian miracidia and Omani cercariae and adult worms were collected previously. We have previously described methods and generation of exome sequence from $S$. mansoni samples $(51,52)$. Data were analyzed the same way as described in Chevalier et al. (52). Code is available in Jupyter notebook and scripts associated (https://github.com/fdchevalier/PZQ-R_field). 
To confirm the presence of the variants in Sm.TRPMPZQ gene from our exome-sequenced natural S. mansoni parasites (when read depth was $<10$ reads), we performed Sanger re-sequencing of eight $S m$.TRPMPZQ exons (i.e. exon 3, 4, 23, 25, 27, 29 and 34) where either nonsense mutations (leading to truncated protein) or nonsynonymous mutation located close to the PZQ binding site (21) were identified. Primers and conditions are listed in Table S4.

\section{Sanger sequencing analysis}

We scored variants using PolyPhred software (v6.18) (Nickerson et al., 1997) which relies on Phred (v0.020425.c), Phrap (v0.990319), and Consed (v29.0) software, analyzing each exon independently. We identified single nucleotide polymorphisms using a minimum phred quality score (-q) of 25 , a minimum genotype score (-score) of 70, and a reference sequence of the $S m$.TRPM $P Z Q$ gene from the chromosome 3 of S. mansoni reference genome (schistosoma_mansoni.PRJEA36577.WBPS14). Variant sites were labeled as non-reference alleles if they differed from the reference sequence. We identified insertion/deletion (indel) polymorphisms using a minimum phred quality score (-q) of 25, a minimum genotype score (-iscore) of 70. Polymorphisms were visually validated using Consed. All the sequences were submitted to GenBank (GenBank accession no KU951903-KU952091). These analyses are available in the Jupyter notebook and associated scripts (https://github.com/fdchevalier/PZQ-R_DNA).

\section{$\underline{\text { Statistical analysis }}$}

All statistical analyzes and graphs were performed using R software (v3.5.1) (74). We used the drc package from $\mathrm{R}$ to analyze all our dose-response datasets using a four-parameter log-logistic function to fit curves. We used the Readqpcr and Normqpcr packages to analyze all our RT-qPCR datasets, using the efficiency $y^{\Delta \Delta \mathrm{Ct}}$ method. When data were not normally distributed (Shapiro test, $p<0.05$ ), we compared results with nonparametric tests: Chi-square test, Kruskal-Wallis test followed by pairwise Wilcoxon-Mann-Whitney posthoc test or a simple pairwise comparison Wilcoxon-Mann-Whitney test. When data followed a normal 


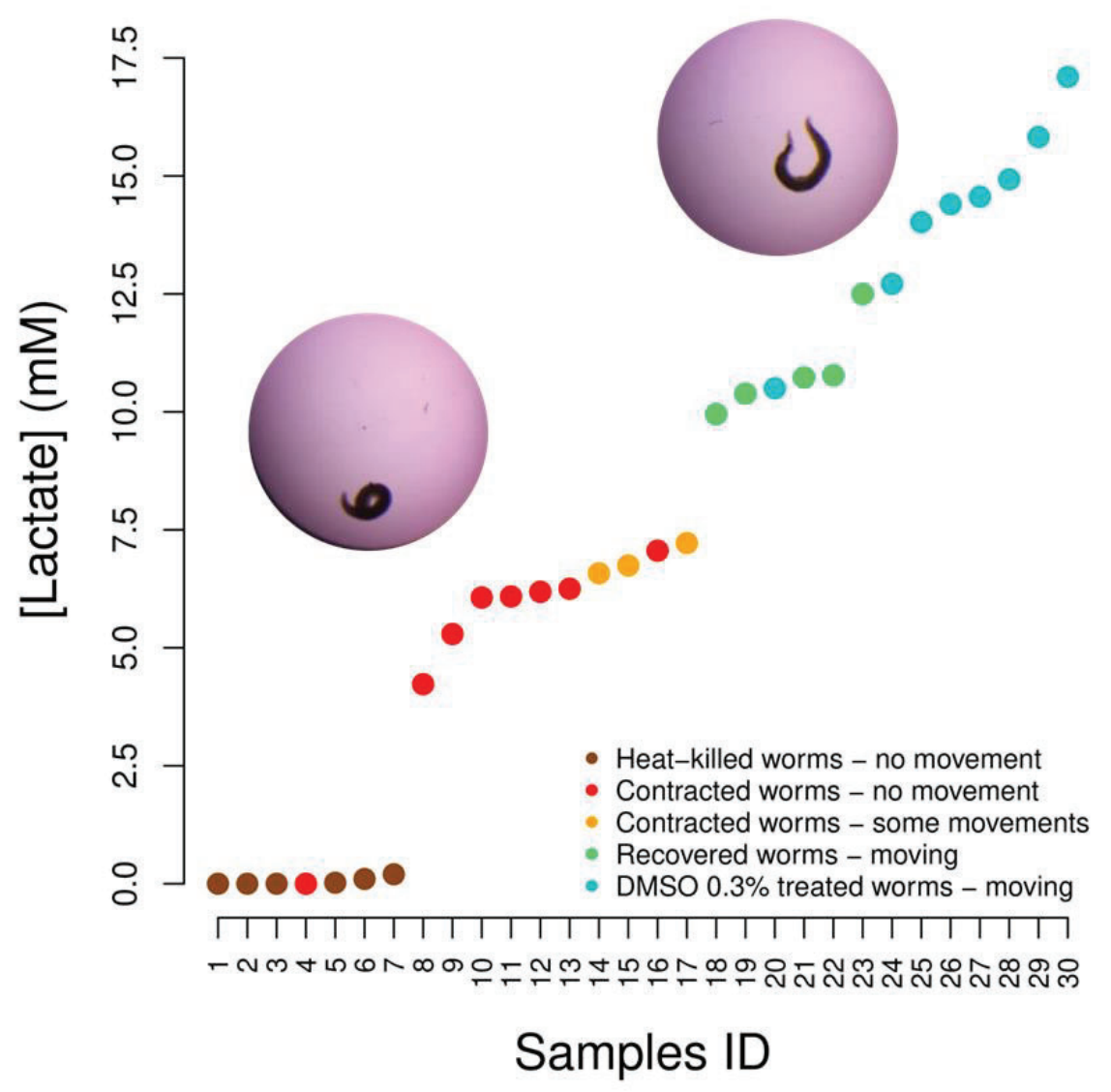

Fig. S1. Development of a lactate assay for assaying worm recovery. Validation of the L-lactate assay for single male worms and correlation with their microscopic appearance and ability to regain movement after PZQ treatment $(24.3 \mu \mathrm{g} / \mathrm{mL})$. PZQ-S worms (contracted), that remain contracted after PZQ treatment produce significantly less amount of lactate released in the media compared to PZQ-R (recovered and motile) worms (Wilcoxon test, $\mathrm{p}=0.0015 ; N=30$ worms). 


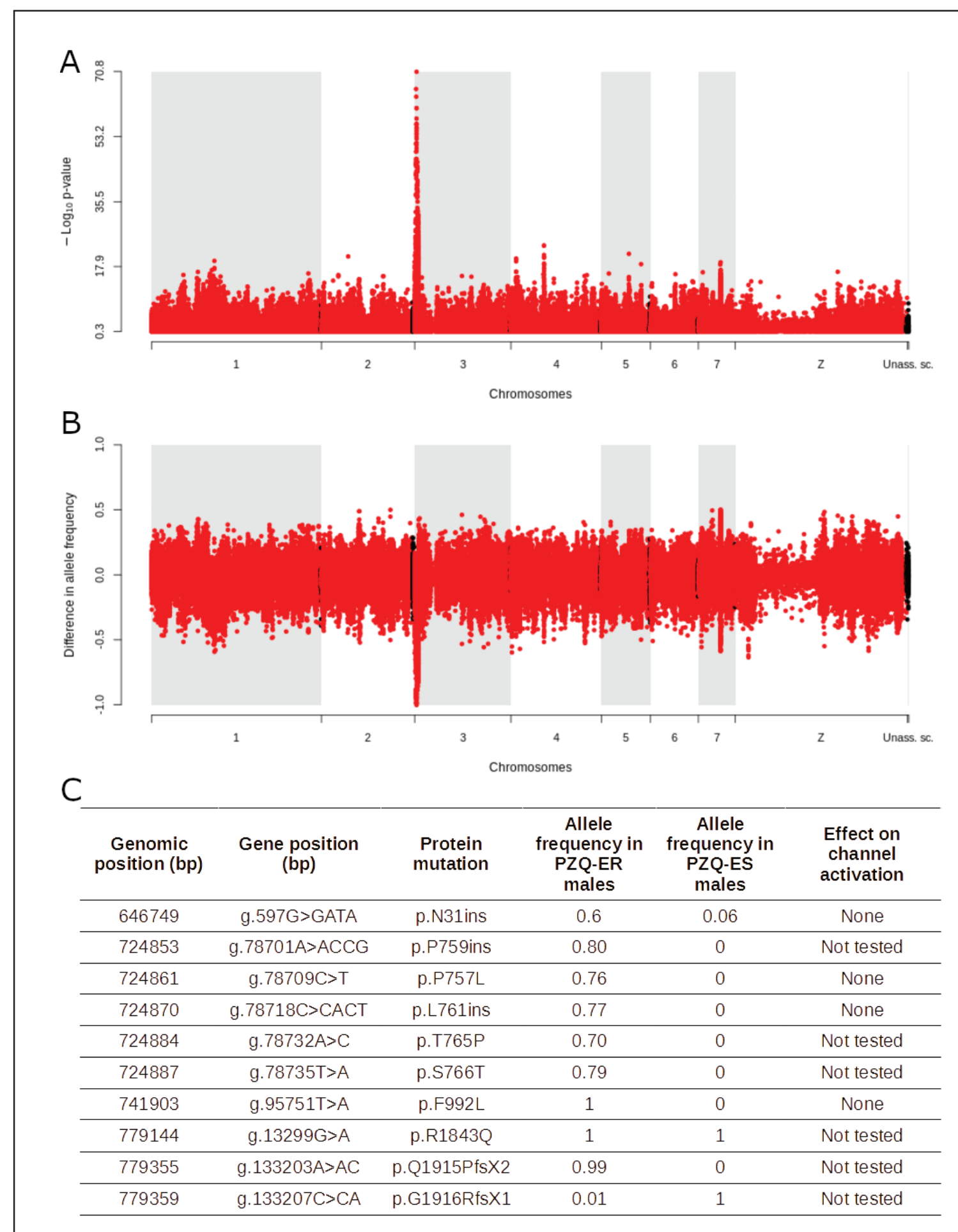

Fig. S2. Validation of marker-assisted selection of SmLE-PZQ-ER and ES using Next

Generation Sequencing (NGS). SmLE-PZQ-ER and ES differed only at the locus linked to PZQ resistance (A). Alternatively fixed allele were fixed for alternative alleles at the $\operatorname{Sm} . T R P M_{P Z Q^{-}}$ 741987C SNP genotyped (B; 1: fixed in ES, -1 fixed in ER), but showed similar allele frequencies across the rest of the genome. Segregating mutations between ER and ES are shown in table (C). 


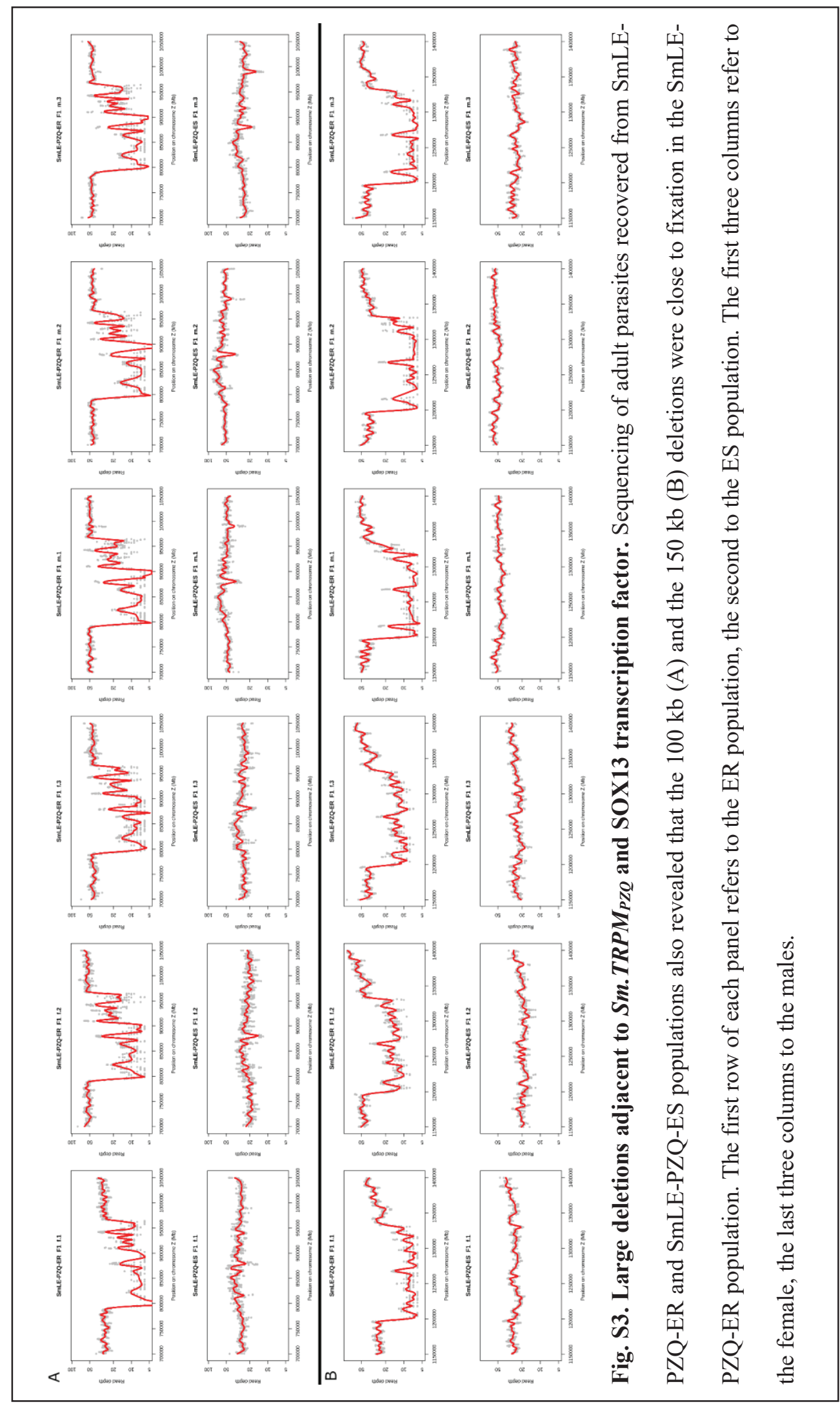

ฮั ำ 

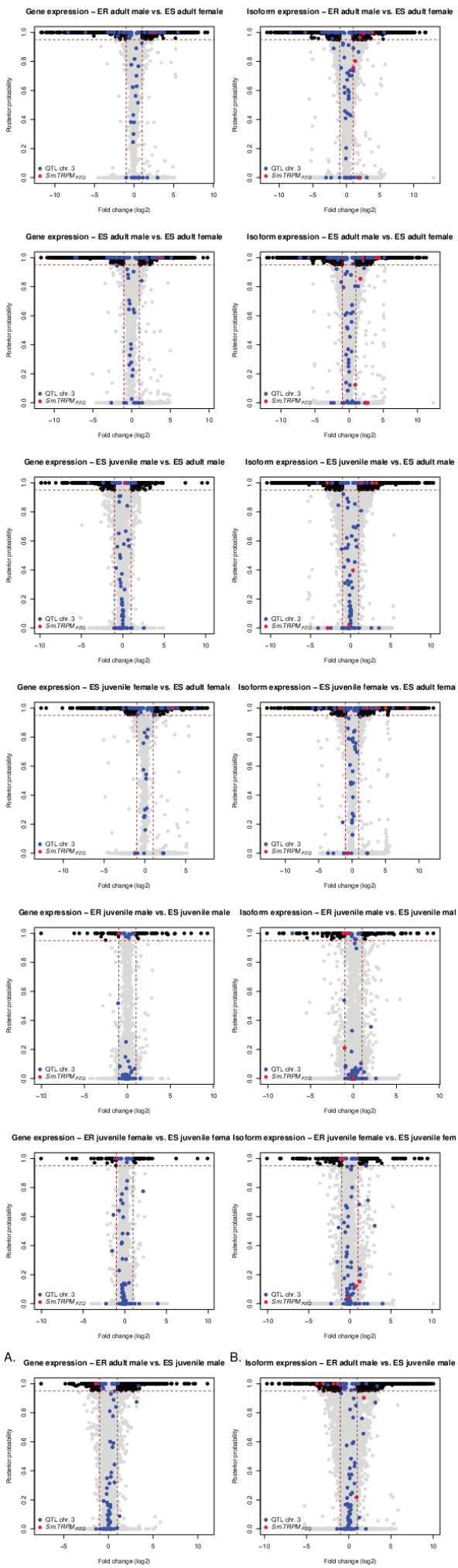

Fig. S4. Detailed genes and isoforms expression in SmLE-PZQ-ER and SmLE-PZQ-ES parasites. Comparison of genes and isoforms expression between SmLE-PZQ-ER and ES parasites for each sex (i.e. male and female) and each parasite stage (i.e. adult worm and juvenile worm) 
A.

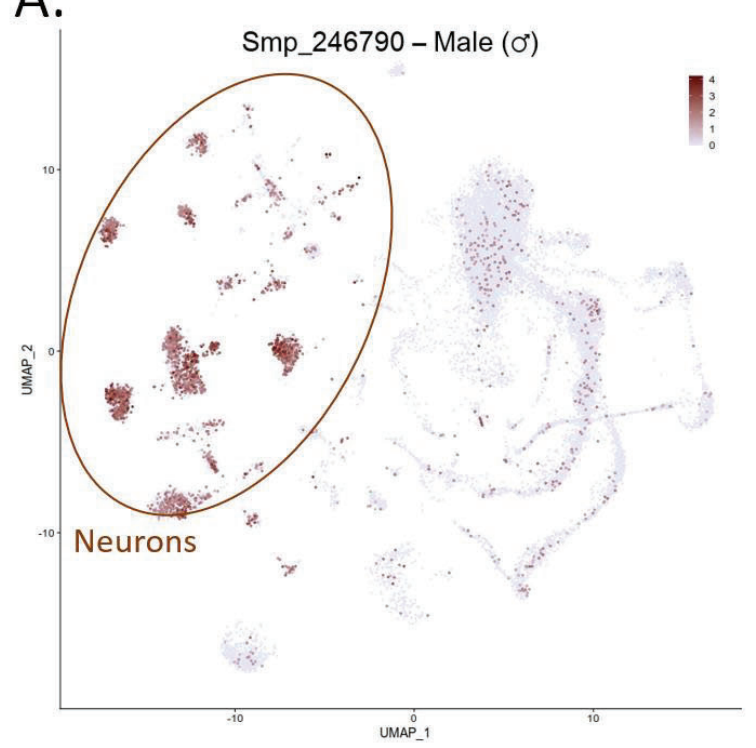

C.

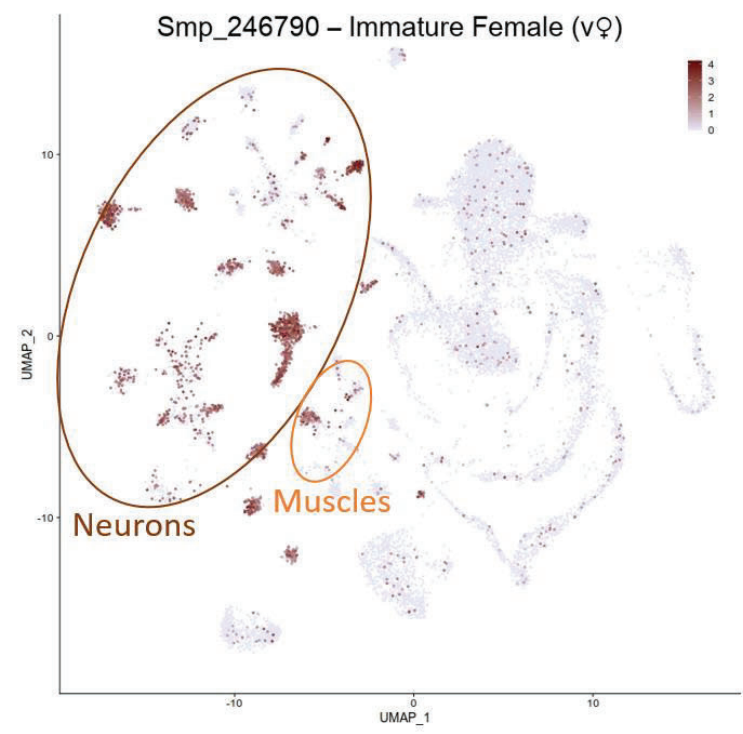

B.

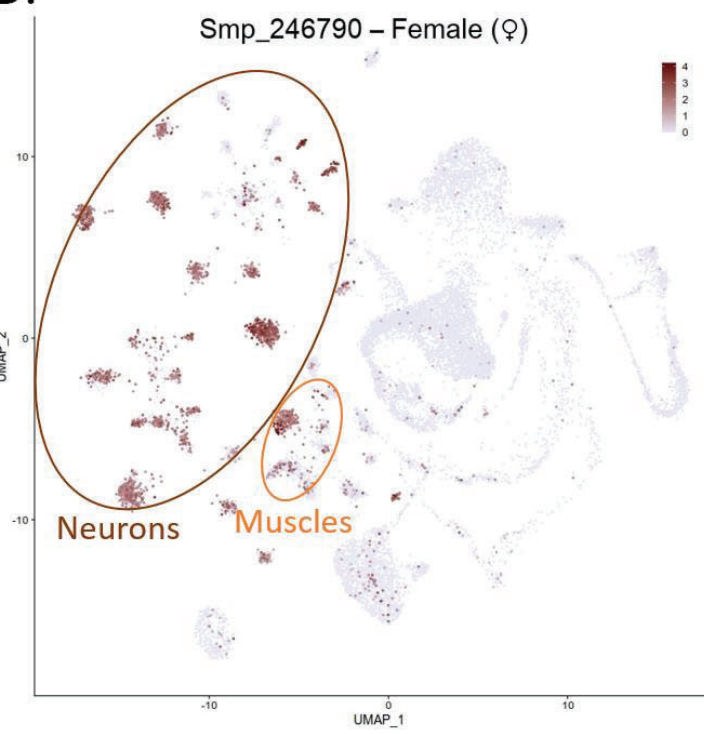

D.

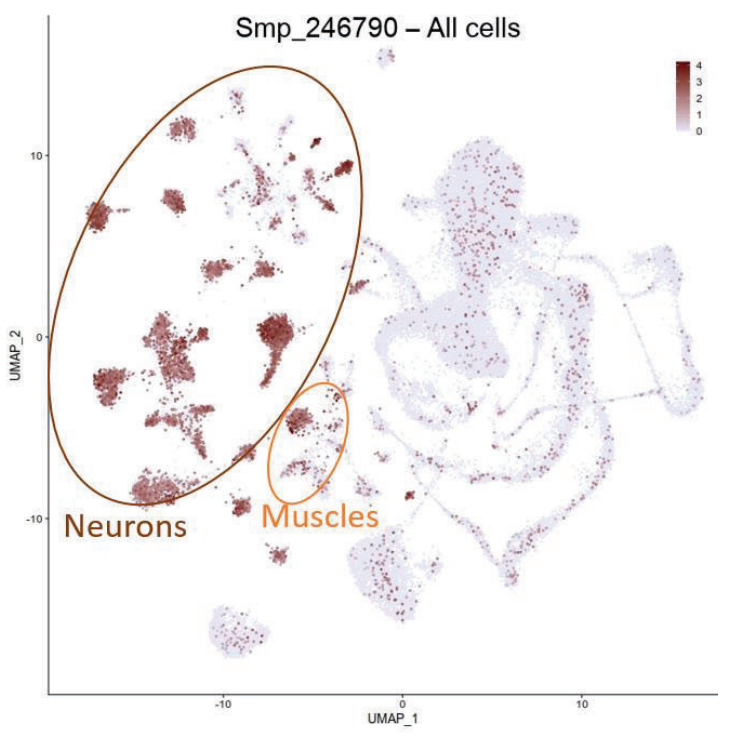

Fig. S5. Cellular localization of $\boldsymbol{S m}$.TRPMPZQ expression in $\boldsymbol{S}$. mansoni (A) adult male, (B) adult female, (C) immature female, (D) overall sex and stages (SchistoCyte Atlas (46)). Sm.TRPM $P Z Q$ gene is essentially expressed in neurons for all sex and stages and is also expressed in muscle cells in females. 

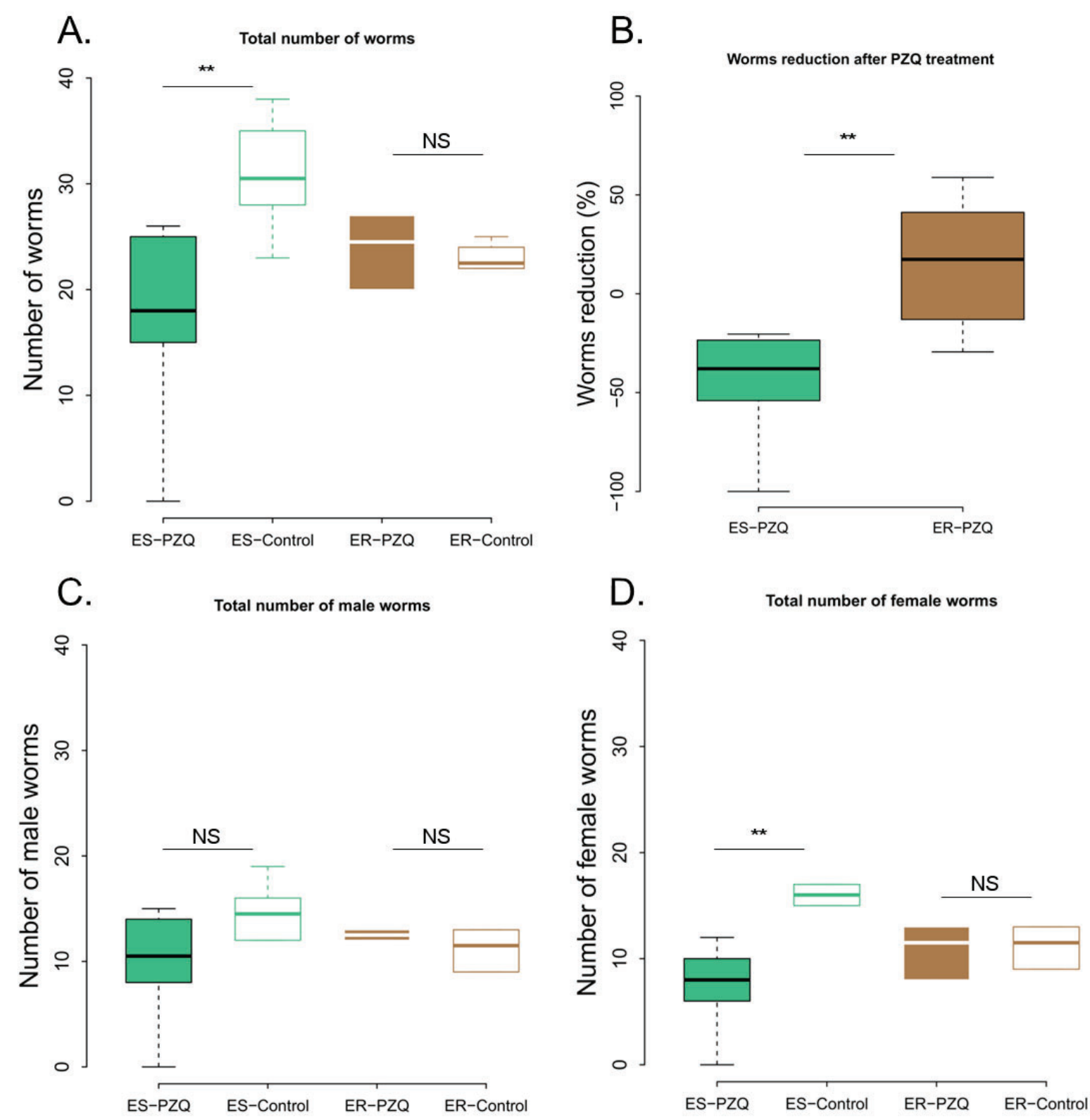

Fig. S6. Impact of in vivo PZQ treatment on SmLE-PZQ-ER and SmLE-PZQ-ES parasites. Balb/c mice were infected with either SmLE-PZQ-ER or SmLE-PZQ-ES parasites populations and treated with $120 \mathrm{mg} / \mathrm{kg}$ of PZQ or DMSO (control group). (A). We observed no significant reduction in worm burden in SmLE-PZQER parasites when comparing PZQ-treated and control (DMSO) treated animals (Wilcoxon test, $\mathrm{p}=0.393$ ). In contrast, we recovered significantly lower numbers of worms from PZQ-treated versus untreated mice infected with the SmLE-PZQ-ES parasite population (Wilcoxon test, $p=0.008$ ). (B). The percent reduction observed was significantly different between the SmLE-PZQ-ES and SmLE-PZQ-ER parasites (Wilcoxon test, $p=0.0129)$. (C). While Interestingly, we did not reach significance for male worms (Wilcoxon test, $p=$ 0.089), (D). we observed a large reduction in numbers of female worms recovered from PZQ-treated SmLEPZQ-ES parasites relative to untreated animals (Wilcoxon test, $\mathrm{p}=0.008)(N=24$ mice -6 mice/group; NS: No significant difference between groups; ${ }^{*} p<0.05$; ** $p \leq 0.02$; *** $p \leq 0.002$ ). 


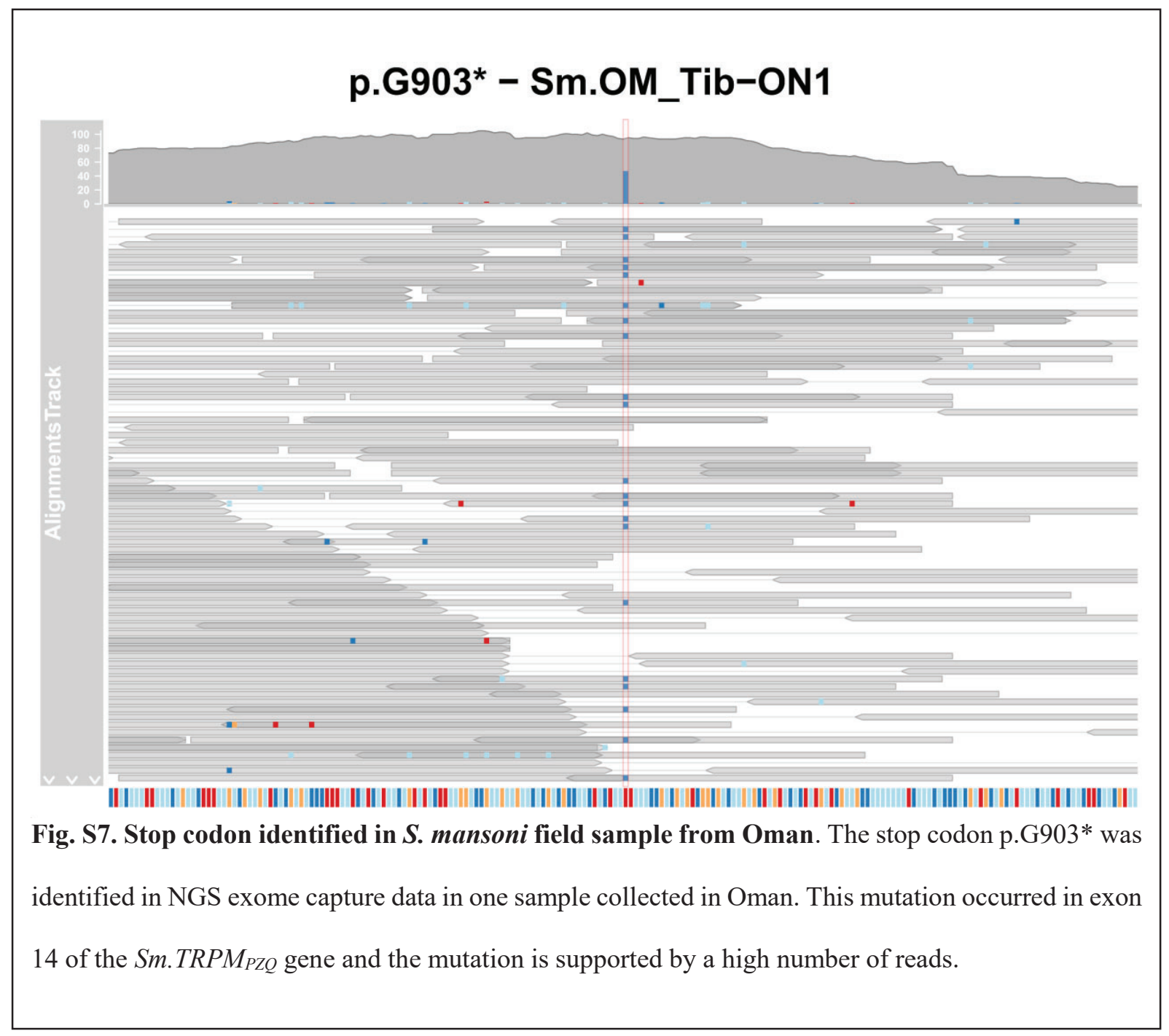


bioRxiv preprint doi: https://doi.org/10.1101/2021.06.09.447779; this version posted June 10, 2021. The copyright holder for this preprint (which was not certified by peer review) is the author/funder, who has granted bioRxiv a license to display the preprint in perpetuity. It is made available under aCC-BY-NC 4.0 International license.

Table S1. Genes in QTL regions on chr 2 and 3.

1219 Separate file 


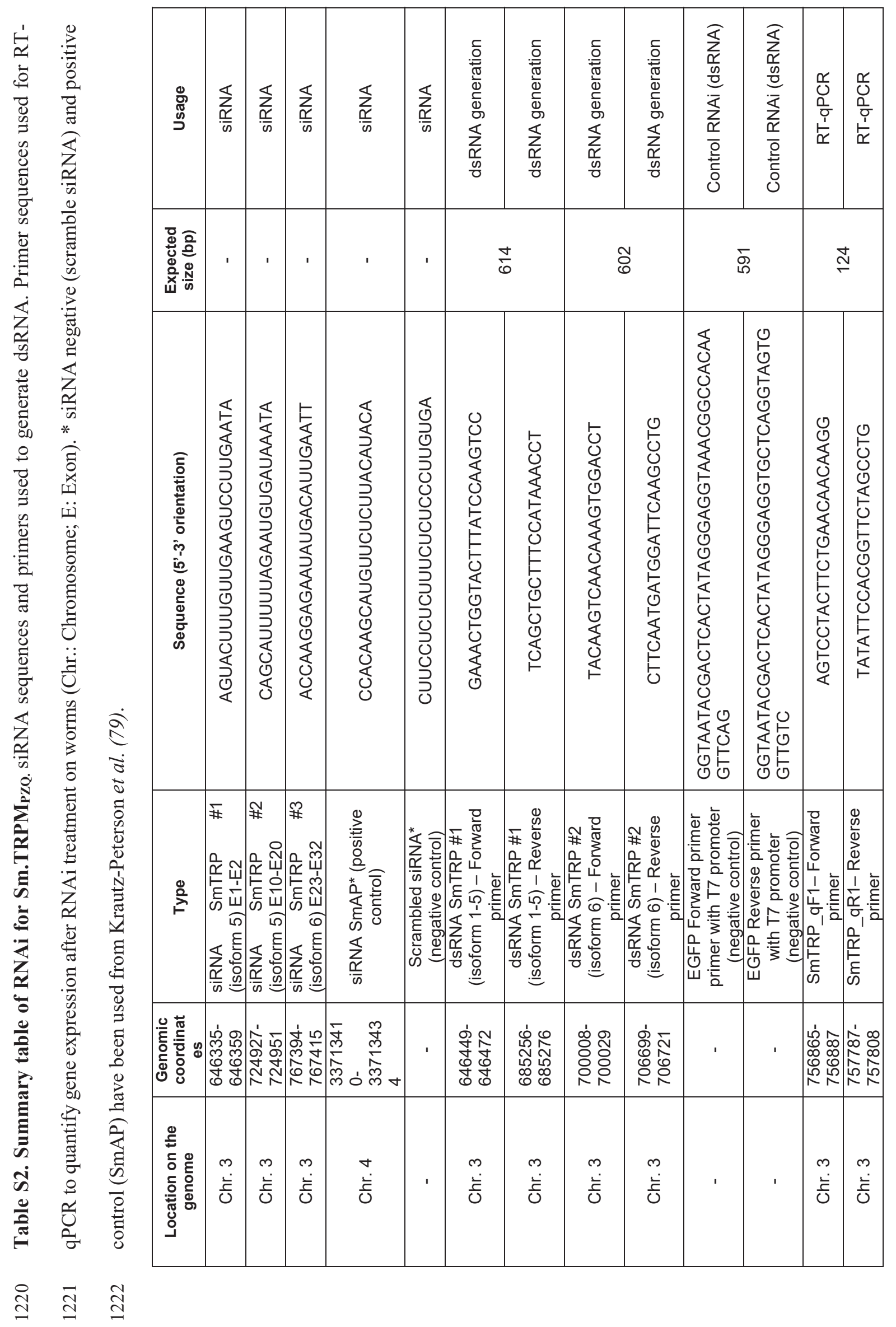




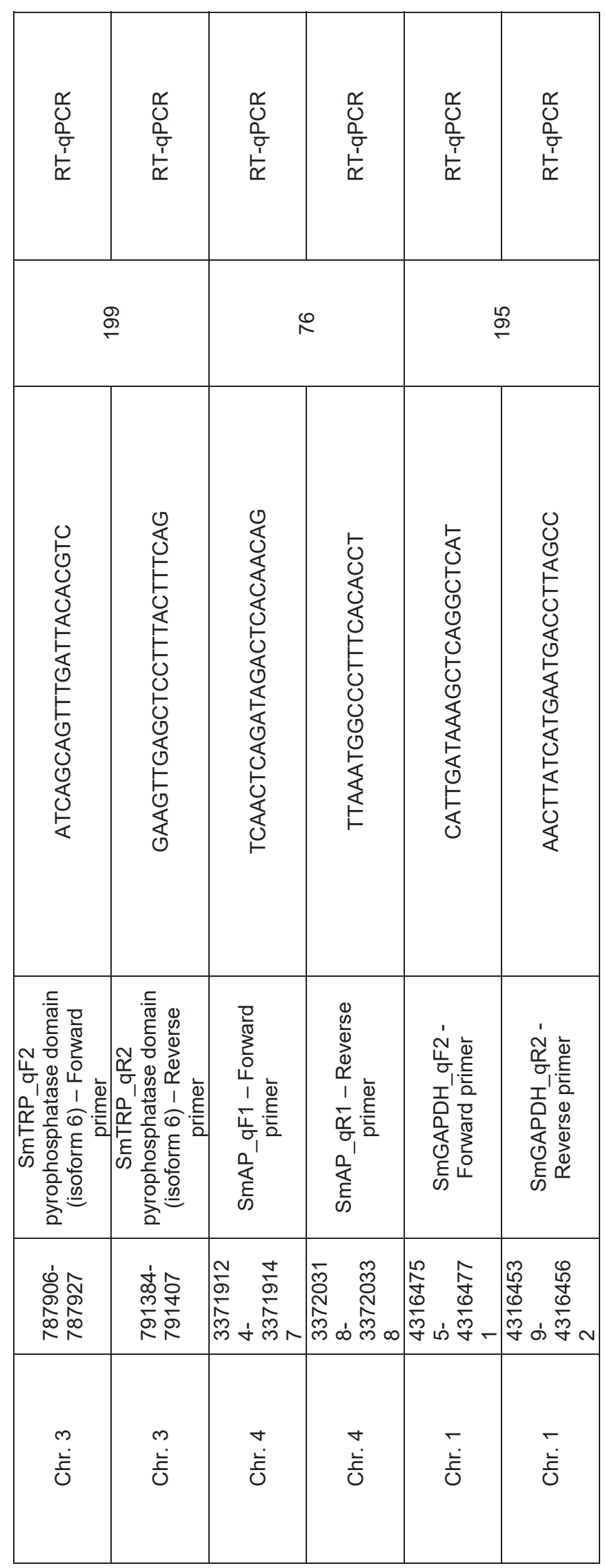


bioRxiv preprint doi: https://doi.org/10.1101/2021.06.09.447779; this version posted June 10, 2021. The copyright holder for this preprint (which was not certified by peer review) is the author/funder, who has granted bioRxiv a license to display the preprint in perpetuity. It is made available under aCC-BY-NC 4.0 International license.

Table S3. Mutations present in Sm.TRPMPZQ in natural schistosome populations from 3 African countries (Senegal, Niger, Tanzania), the Middle East (Oman) and South America (Brazil). 


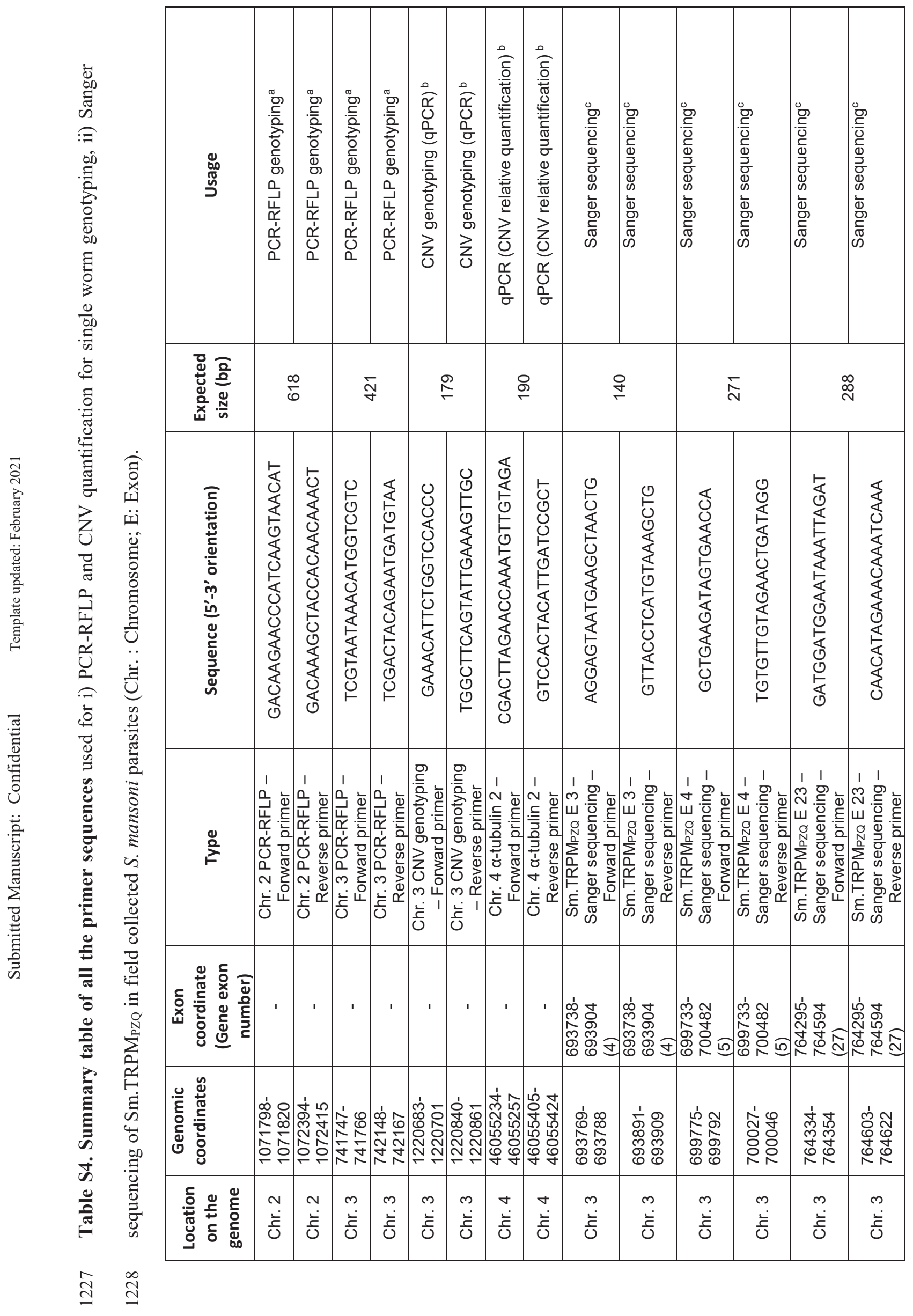




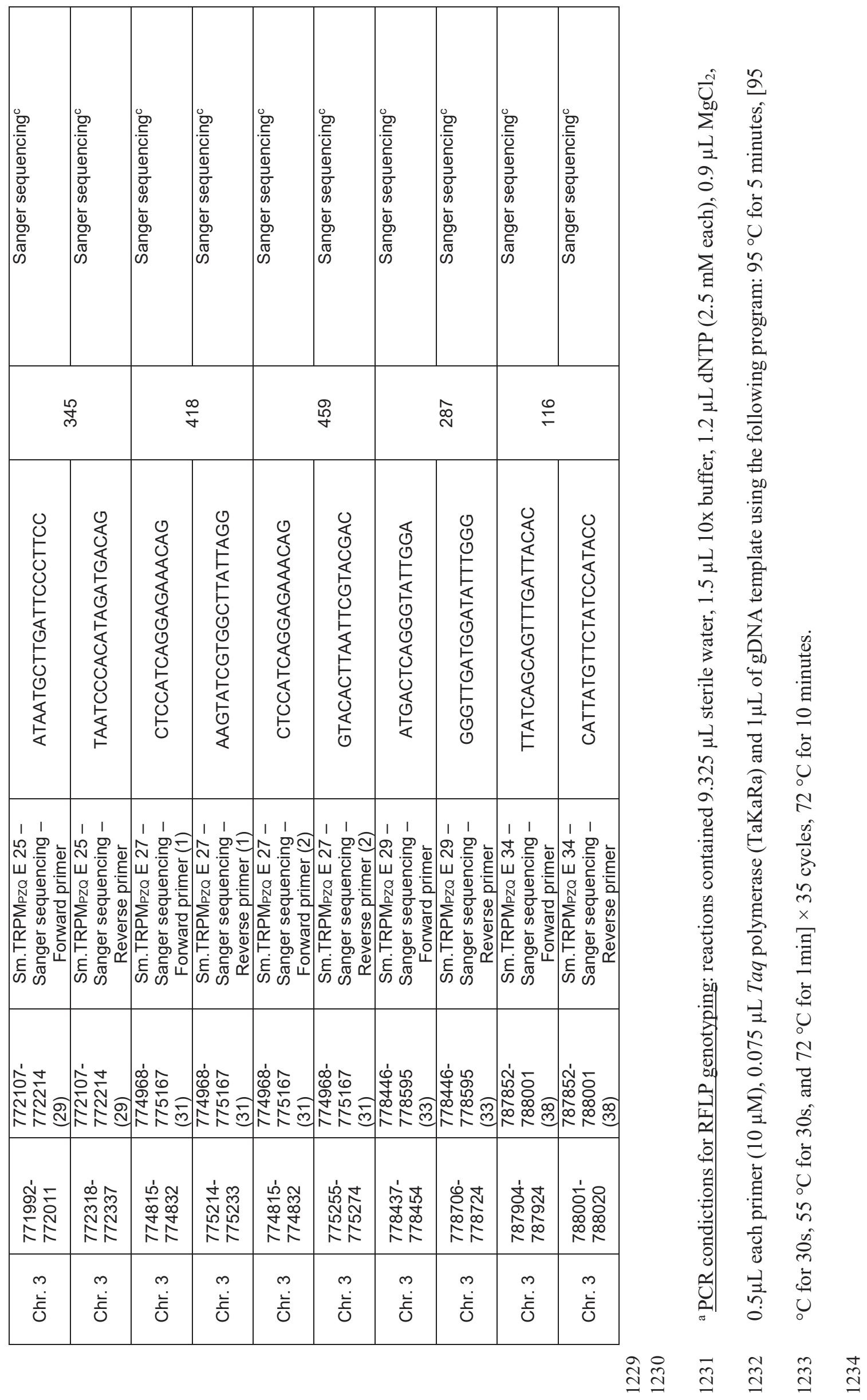




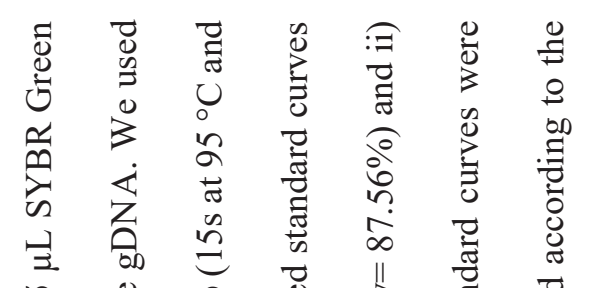

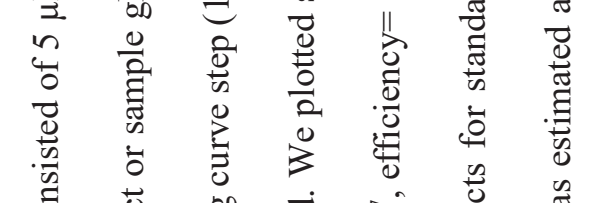

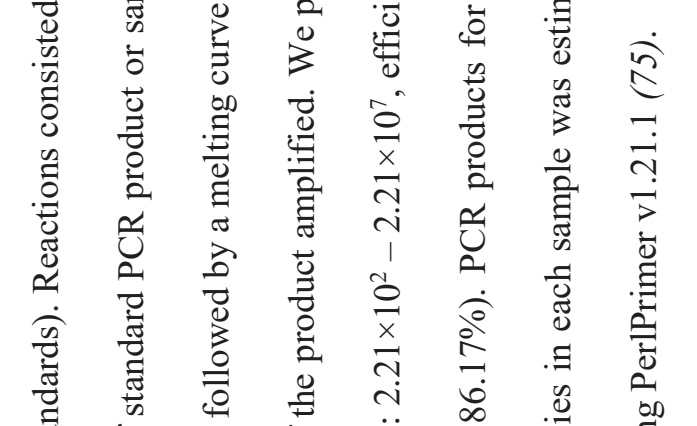

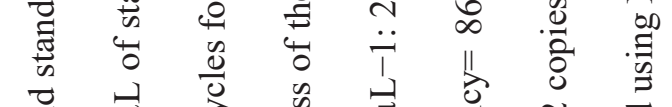

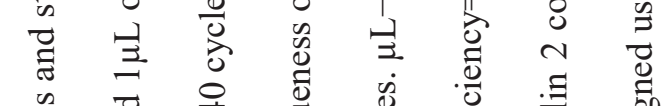

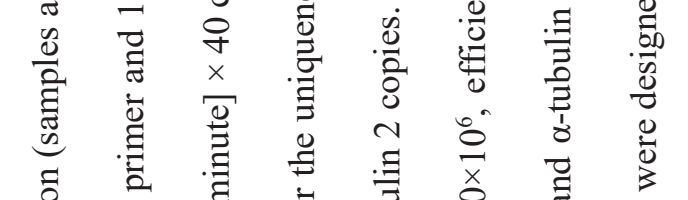

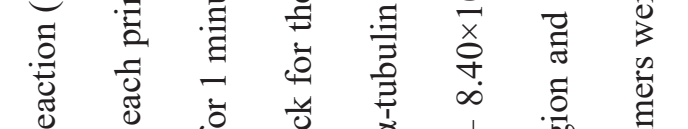

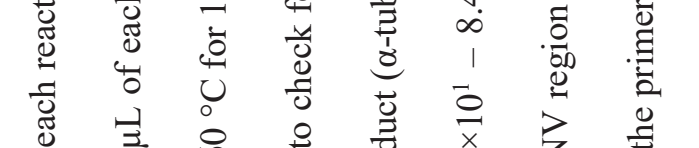

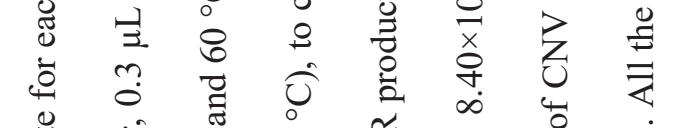

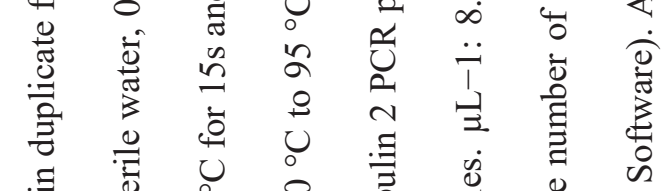

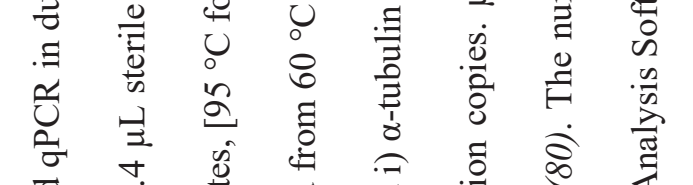

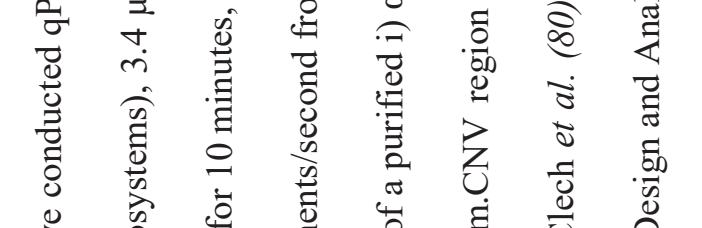

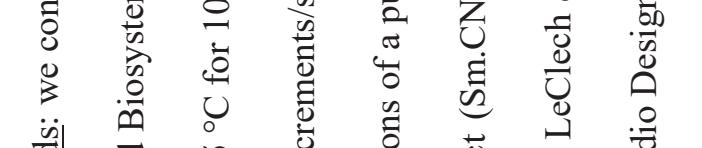

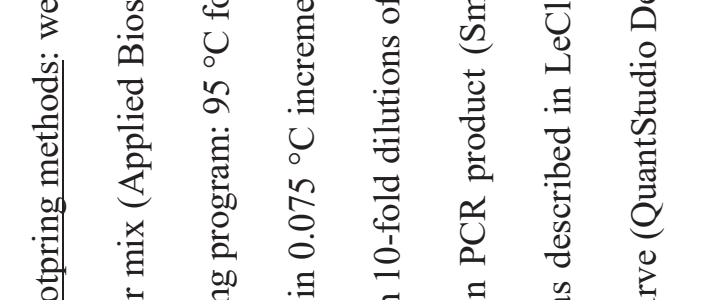

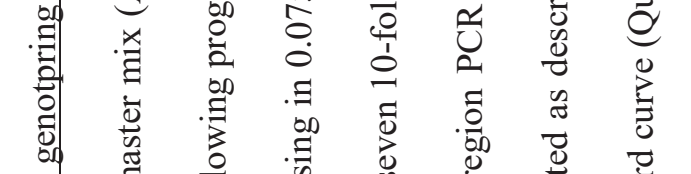

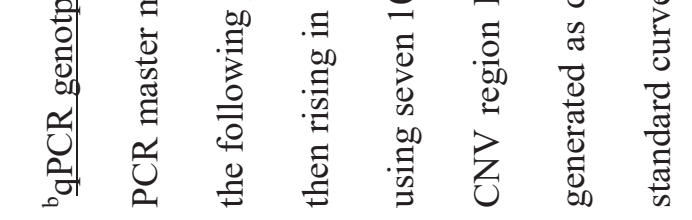

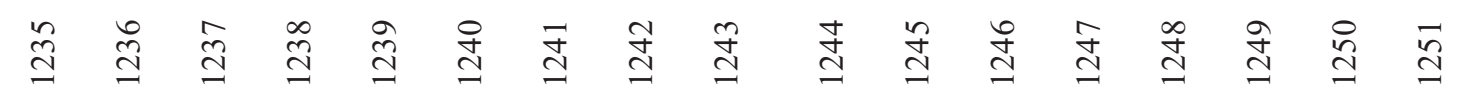

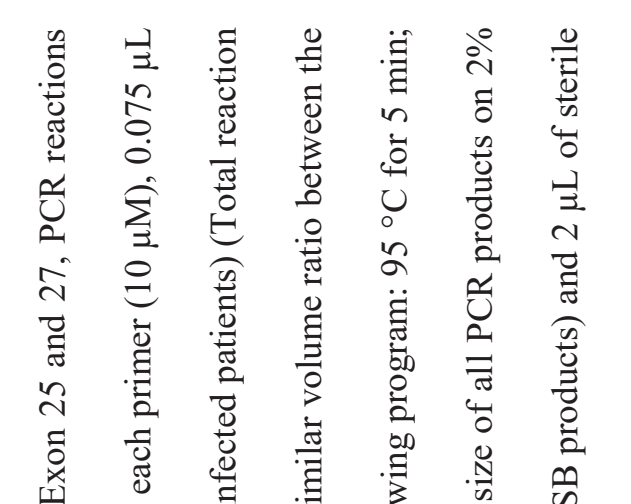

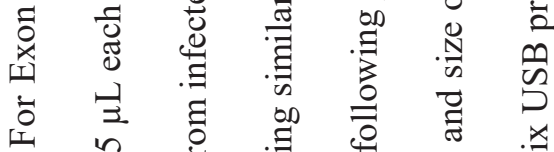

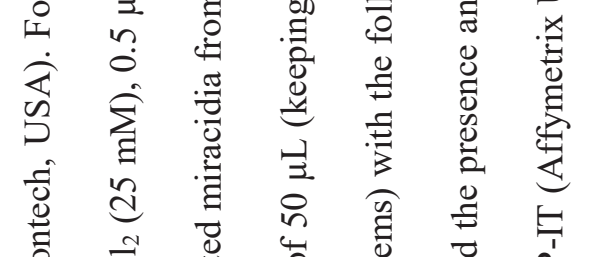

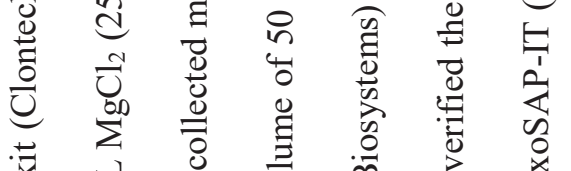

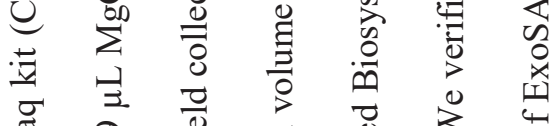

弯

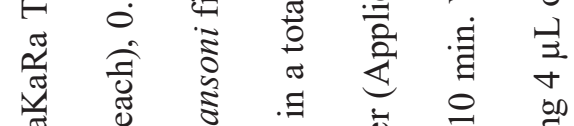

焉

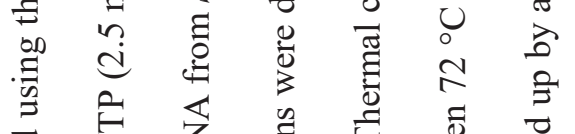

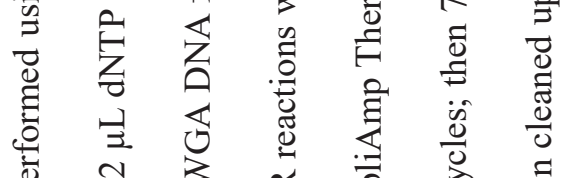

离

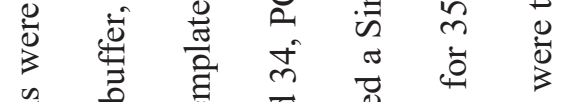
苟 ij

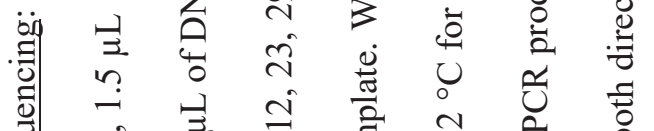

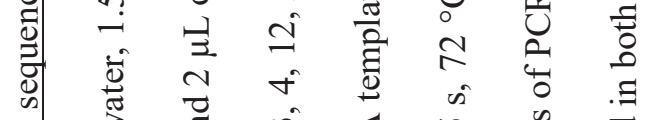

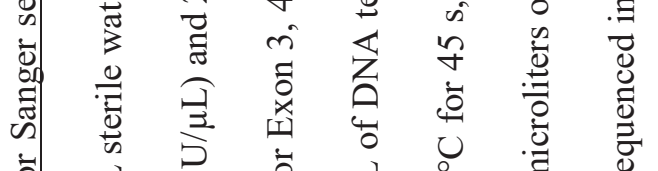

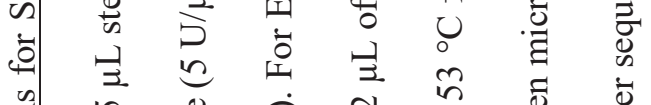

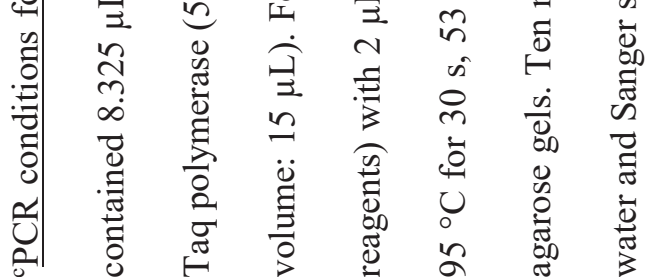

